\title{
Constraining Dark Energy by Combining Cluster Counts and Shear-Shear Correlations in a Weak Lensing Survey
}

\author{
Wenjuan Fang ${ }^{1}$ and Zoltán Haiman ${ }^{2}$ \\ ${ }^{1}$ Department of Physics, Columbia University, New York, NY 10027, USA \\ ${ }^{2}$ Department of Astronomy, Columbia University, New York, NY 10027, USA
}

(Dated: August 4, 2018)

\begin{abstract}
We study the potential of a large future weak-lensing survey to constrain dark energy properties by using both the number counts of detected galaxy clusters (sensitive primarily to density fluctuations on small scales) and tomographic shear-shear correlations (restricted to large scales). We use the Fisher matrix formalism, assume a flat universe and parameterize the equation of state of dark energy by $w(a)=w_{0}+w_{a}(1-a)$, to forecast the expected statistical errors from either observable, and from their combination. We show that the covariance between these two observables is small, and argue that therefore they can be regarded as independent constraints. We find that when the number counts and the shear-shear correlations (on angular scales $\ell \leq 1000$ ) are combined, an LSST (Large Synoptic Survey Telescope)-like survey can yield statistical errors on $\Omega_{\mathrm{DE}}, w_{0}, w_{a}$ as tight as $0.003,0.03,0.1$. These values are a factor of $2-25$ better than using either observable alone. The results are also about a factor of two better than those from combining number counts of galaxy clusters and their power spectrum.
\end{abstract}

\section{INTRODUCTION}

The existence of dark energy is strongly indicated by the relatively dim appearance of distant supernovae [1, 2], by the shortfall of the matter density to make the universe spatially flat (e.g. [3]), and by recent, accurate measurements of cosmic microwave background (CMB) anisotropy [4, 5]. This newly discovered form of energy constitutes about $2 / 3$ of the total energy density of the universe, and is known to have negative pressure and a nearly uniform spatial distribution. Several competing theoretical models have been proposed to explain dark energy (see, e.g., [6] for a list of references). While current observational data cannot distinguish among these proposals, it is hoped that future observations, which will reach higher precision, can constrain models, and clarify the nature of dark energy.

In this paper, we explore one of the methods to constrain dark energy parameters in the future: using the weak gravitational lensing (WL) distortion of distant galaxies in a large survey of the sky. The light from distant galaxies is deflected by the foreground gravitational field, causing small but statistically coherent distortions in the observed shapes of the galaxies. This socalled weak-lensing shear signal can be observed, in principle, for a large number of galaxies, and used to infer the foreground gravitational field or almost equivalently, the mass distribution (see recent reviews by, e.g., [7, 8]) ).

Several previous studies have examined the constraints that can be placed on dark energy from large weaklensing surveys. The most commonly proposed method is to utilize statistical properties of the two-dimensional shear field directly, such as its power spectrum (or, equivalently, the shear-shear correlation function). With additional photometric redshift information, which future wide-field multi-color surveys are expected to provide, the background galaxies can be grouped into different redshift bins. Statistics done within each bin and among different bins can recover some of the information from the third (radial) dimension. Such a "tomographic" study of the foreground density fluctuations provides an additional handle on dark energy, through the effect of dark energy on the recent $(0<z \lesssim 1)$ growth of dark matter perturbations and the expansion history of the universe (e.g. [9, 10, 11, 12, 13, 14, 15]). In particular, Song \& Knox [15] have evaluated the statistical constraints from tomographic shear-shear correlations that are expected to be available from the Large Synoptic Survey Telescope (LSST).

A complementary method is to utilize statistical properties of the peaks of the shear field. This method, however, does not lend itself to straightforward mathematical analysis, and has been relatively much less well explored (e.g. [16, 17]) On the other hand, there is an increasingly better correspondence between higher$\sigma$ shear peaks and discrete, massive virialized objects -galaxy clusters- in the foreground [18, 19]. To the extent that this correspondence can be quantified abinitio in numerical simulations, one can use the shearselected cluster sample, including their abundance evolution and power spectrum, to constrain dark energy properties (e.g. [20, 21]). In general, the abundance evolution of galaxy clusters can place strong constraints on dark energy parameters, because it is exponentially sensitive to the growth rate of matter fluctuations. Several studies have explored constraints expected from future surveys that would detect clusters through their X-ray emission or the Sunyaev-Zeldovich effect (e.g. [22, 23, 24, 25]. An attractive feature of utilizing weak lensing signal to detect clusters is that it directly probes the total mass of the cluster. In particular, Wang et al. 20] have evaluated the statistical constraints from the galaxy cluster sample that is expected to be available from LSST.

Once the data from a wide-field WL survey, such as LSST [26], or a smaller pre-cursor mission, such as Pan- 
Starr [27] has been collected, it will be logical to try to extract information on dark energy properties from both the shear-shear correlations and cluster abundance, since both pieces of information will be available in the same data-set. The goal of the present paper is to quantify the improvement on dark energy constraints when these two pieces of information are combined.

Ideally, one would pose more ambitious questions, such as: what is the maximum information one can obtain on dark energy parameters, given the effect of dark energy on the full non-linear shear field? In particular, it is not clear whether either statistic (shear-shear correlations or cluster counts), in fact, captures a significant fraction of the available information. Nonetheless, in this paper, we contend ourselves to answering the much simpler question above. Furthermore, for simplicity, we will use the shear-shear correlation function only on large angular scales $(\ell \leq 1000)$. On large scales, where density fluctuations are in the linear regime, the correlation function contains all the information about the density field. On smaller scales, non-linear evolution introduces significant non-Gaussianity. We will argue below that once cluster counts are taken into account, the smallscale shear-shear correlations offer no significant additional information. In this paper, we focus on evaluating constraints for a ground-based survey, such as LSST. We parameterize dark energy by its bulk equation of state $w=\langle P\rangle /\langle\rho\rangle$ [28], and allow $w$ to evolve linearly with the cosmic scale-factor $a$ as $w(a)=w_{0}+w_{a}(1-a)$.

This paper is organized as follows. In $\S$ II, we describe our calculational methods, which closely follow previous Fisher matrix analyses, but include an additional discussion of covariance. Our results are presented in $\S$ III. and discussed, along with various caveats, in $\S$ IV. Finally, in $\S \nabla$ we offer our conclusions and summarize the implications of this work.

\section{CALCULATIONAL METHODS}

\section{A. Cluster Number Counts}

We closely follow ref. [20], and consider a sample of shear-selected clusters with specifications motivated by LSST. In particular, we assume the sample covers the redshift range of $0.1 \leq z \leq 1.4$, which is divided into 26 redshift bins of equal size $\Delta z=0.05$. The expected number of clusters in the $i^{\text {th }}$ redshift bin is calculated as

$$
\bar{N}_{i}=\Delta \Omega \Delta z \frac{d^{2} V}{d z d \Omega}\left(z_{i}\right) \int_{M_{\min }\left(z_{i}\right)}^{\infty} \frac{d n}{d M}\left(M, z_{i}\right) d M
$$

where $z_{i}$ is the central redshift for the $i^{\text {th }}$ redshift bin, $\Delta \Omega$ is the solid angle covered by the survey, which for LSST, we take to be $18,000 \mathrm{deg}^{2}, d^{2} V / d z d \Omega$ is the comoving volume element, $M_{\min }$ is the detection threshold mass for clusters, and $\frac{d n}{d M}$ is the cluster mass function.
We use the fitting formula given by Jenkins et al. [29] for the cluster mass function,

$$
\begin{aligned}
\frac{d n}{d M}(M, z)= & 0.301 \frac{\rho_{m}}{M} \frac{d \ln \sigma^{-1}(M, z)}{d M} \\
& \times \exp \left(-\left|\ln \sigma^{-1}(M, z)+0.64\right|^{3.82}\right)
\end{aligned}
$$

where $\rho_{m}$ is the present-day matter density, $\sigma(M, z)$ is the amplitude of the linear matter fluctuations at redshift $z$, smoothed by a top hat window function whose scale is such that the enclosed mass at the mean density $\rho_{m}$ is $M$. This formula is based on the identification of dark matter halos as spherical regions that have a mean overdensity of 180 with respect to the background matter density at the time of identification.

In determining the mass threshold for detecting a cluster at redshift z, $M_{\min }(z)$, we follow NFW [30], and model the density profile of galaxy clusters with the self-similar function

$$
\rho_{\mathrm{NFW}}(r)=\frac{\rho_{s}}{\left(r / r_{s}\right)\left[1+\left(r / r_{s}\right)\right]^{2}},
$$

where $r$ is the radius from the cluster center, and $r_{s}, \rho_{s}$ are some characteristic radius and density. In [30], the density profile is truncated at $r_{200}$, inside which the mean overdensity with respect to the critical density of the universe at redshift $z$ is 200 . The outer radius $r_{200}$ is parameterized by the concentration parameter, defined as

$$
c_{\mathrm{NFW}}=\frac{r_{200}}{r_{s}}
$$

and taken to be a constant $c_{\mathrm{NFW}}=5$ in this paper. With the definition of the cluster mass $M_{200}$ as the mass enclosed within $r_{200}$, the cluster's structure $\left(\rho_{s}, r_{s}, r_{200}\right)$ is fully determined by $M_{200}$ and $z$.

We then follow Hamana et al. [18], and use a Gaussian window function to smooth the convergence field induced by the cluster,

$$
\kappa_{G}=\int d^{2} \phi W_{G}(\phi) \kappa(\phi)
$$

$$
W_{G}(\phi)=\frac{1}{\pi \theta_{G}^{2}} \exp \left(-\frac{\phi^{2}}{\theta_{G}^{2}}\right),
$$

where the center of the smoothing kernel is set to that of the cluster. Here $\theta$ is the angular distance from the cluster center, and $\theta_{G}$ is the size of the smoothing aperture, which we choose to be 1 arcmin. Assuming that along the line of sight, there is only one lens at redshift $z$ (i.e., the cluster to be detected), the convergence can be calculated, given a distribution of the background galaxies, as

$$
\kappa(\phi)=\frac{4 \pi G}{c^{2}} \frac{\Sigma(\phi) \chi_{z}}{(1+z)} \frac{\int_{z}^{\infty} d z^{\prime}\left(d n / d z^{\prime}\right)\left(1-\chi_{z} / \chi_{z^{\prime}}\right)}{n_{\mathrm{tot}}} .
$$

Here $\Sigma(\phi)$ is the surface density of the cluster, projected along the line of sight (see [18] for more details), $\chi_{z}$ is 
the comoving radial distance to redshift $z$ (note that we assume a flat universe in this paper), $d n / d z$ is the mean redshift distribution of the surface number density (per steradian) of background galaxies, and $n_{\text {tot }}$ is the mean total surface density.

Throughout this paper, we adopt both $d n / d z$ and $n_{\text {tot }}$ from the ground based survey described in [15]. In particular, those authors assumed $n_{\text {tot }}=65 \operatorname{arcmin}^{-2}$, which may be optimistic by a factor of $\sim$ two (e.g. 21], see also ref.31] for an extended discussion on statistical galaxy shape measurements). In this paper, we are primarily interested in a relative improvement of constraints when two methods are combined, rather than in the absolute constraints. We therefore stick to the optimistic values, to facilitate a comparison with earlier work. We assume the noise on the measured convergence comes from the r.m.s. intrinsic ellipticity of the background galaxies, and is given by [32]

$$
\sigma_{\text {noise }}^{2}=\frac{\sigma_{\epsilon}^{2}}{4} \frac{1}{2 \pi \theta_{G}^{2} n_{\text {tot }}} .
$$

Here $\sigma_{\epsilon}$ is the weighted average of the r.m.s. intrinsic ellipticity per component of the galaxies, given by

$$
\sigma_{\epsilon}^{2}=\frac{\int_{0}^{\infty} d z(d n / d z) \sigma_{\epsilon}^{2}(z)}{n_{\mathrm{tot}}}
$$

where we follow [15], and take $\sigma_{\epsilon}(z)$ to be

$$
\sigma_{\epsilon}(z)=0.3+0.07 z .
$$

Finally, we set the threshold convergence $\kappa_{\text {th }}$ to be 4.5 times the noise. Setting $\kappa_{G}$ in equation (5) equal to $4.5 \sigma_{\text {noise }}$ yields an implicit equation for $M_{200}(z)$. To be consistent with the halo mass defined for the Jenkins et al. fitting formula for the cluster mass function, we extend the NFW density profile for a cluster with mass $M_{200}(z)$ outward to a radius so that the mean interior density is 180 times that of the background matter density at redshift $z$. The mass enclosed within this radius is adopted as $M_{\min }(z)$ in equation (1).

In the mock survey defined above (and with the fiducial cosmological parameters defined below), we find that the limiting halo mass is $\sim(0.6-4) \times 10^{14} \mathrm{M}_{\odot}$, depending on redshift. The smallest halos therefore correspond to small groups, containing, on average, $\sim(10-60)$ galaxies above the absolute $r$ magnitude $M_{r} \lesssim-20$ threshold for the Sloan Digital Sky Survey (see Figure 3 in ref.[33]). Note that LSST will detect galaxies to a much greater depth, which may increase the number of detectable member galaxies (although this is unclear; see, e.g., Figure 3 in ref. [34] that shows no increase).

The total number of clusters in the survey down to this mass threshold is $N_{\text {total }}=276,794$. This is somewhat larger than the number in our previous study [20]. The reason for the increase is that in the previous paper, we excluded background galaxies at $z>2.5$, whereas here we include them (and both distributions were normalized to have the same $\left.n_{\text {tot }}\right)$. As a result, we find here an increase in the convergence produced by a given foreground galaxy cluster. Two additional, smaller differences are that here we adopt a smoothing filter size of 1 arcmin (rather than 0.5 arcmin), and that we use a slightly higher, redshiftdependent intrinsic ellipticity (given by eq.(10), rather than a fixed value of $0.3 \sqrt{2}$ ).

\section{B. Shear-Shear Correlations}

Here we closely follow ref. 15], and consider the shearshear correlation function in an LSST-like ground based survey. From $z=0$ to $z=3.2$, we divide the background galaxies into 8 equally-spaced redshift bins, and we imagine that the solid angle covered in the survey is probed by pixels of size of $\Omega_{\text {pix }}$. On average, a given pixel will probe $N_{\text {pix }}^{b}$ galaxies in redshift bin ' $b$ ', with $N_{\text {pix }}^{b}=n^{b} \Omega_{\text {pix }}$, where $n^{b}$ is the mean surface density of the galaxies in this redshift bin, and is given by

$$
n^{b}=\int_{z_{\min }^{b}}^{z_{\max }^{b}} d z(d n / d z)
$$

with $z_{\min }^{b}$ and $z_{\max }^{b}$ the edges of this redshift bin.

The detected shear from these galaxies depends both on the lensing shear signal and on their intrinsic ellipticity

$$
\begin{aligned}
& \gamma_{1, \text { pix }}^{b}=\gamma_{1, \text { lens }}+\gamma_{1, \text { int }}=\frac{1}{N_{\text {pix }}^{b}} \sum_{i=1}^{N_{\text {pix }}^{b}}\left(\frac{\epsilon_{+i, \text { lens }}}{2}+\frac{\epsilon_{+i, \text { int }}}{2}\right)(12) \\
& \gamma_{2, \text { pix }}^{b}=\gamma_{2, \text { lens }}+\gamma_{2, \text { int }}=\frac{1}{N_{\text {pix }}^{b}} \sum_{i=1}^{N_{\text {pix }}^{b}}\left(\frac{\epsilon_{\times i, \text { lens }}}{2}+\frac{\epsilon_{\times i, \text { int }}}{2}\right)(13)
\end{aligned}
$$

where, $\gamma_{1} \gamma_{2}$ are the two independent components of the shear field, $\epsilon_{+} \epsilon_{\times}$are the two components of the galaxy ellipticity. As before, the intrinsic ellipticity is the only source of noise we consider in this paper for the shearshear correlations. Furthermore, we assume that there is no correlation between intrinsic ellipticity of different galaxies. In this case, $\gamma_{1, \text { int }}$, when averaged over large enough realizations of the intrinsic ellipticity of these $N_{\text {pix }}^{b}$ galaxies, will have a variance of

$$
\begin{aligned}
\left\langle\gamma_{1, \text { int }}^{2}\right\rangle & =\frac{1}{4\left(N_{\text {pix }}^{b}\right)^{2}}\left\langle\sum_{i} \epsilon_{+i, \text { int }}^{2}\right\rangle \\
& =\frac{\int_{z_{\min }^{b}}^{z_{\max }^{b}} d z(d n / d z)\left\langle\epsilon_{+, \text {int }}^{2}(z)\right\rangle}{4 N_{\text {pix }}^{b} n^{b}},
\end{aligned}
$$

A similar expression holds for $\gamma_{2, \text { int }}$. Following 15] again, we assume that the r.m.s. intrinsic ellipticity varies with redshift as

$$
\left\langle\epsilon_{+, \text {int }}^{2}(z)\right\rangle=\left\langle\epsilon_{\times, \text {int }}^{2}(z)\right\rangle=\sigma_{\epsilon}^{2}(z),
$$


where $\sigma_{\epsilon}(z)$ is given by equation (10).

We assume further that there is no correlation between the lensing signal and noise, and expand the shear fields in terms of spherical harmonics. The covariance matrix $\mathbf{C}^{\gamma \gamma}$ of the expansion coefficients in the E mode can then be written as the sum of two matrices $\mathbf{S}^{\gamma \gamma}$ (signal) and $\mathbf{N}^{\gamma \gamma}$ (noise), whose elements are

$$
\begin{aligned}
S_{\ell m, \ell^{\prime} m^{\prime}}^{b, b^{\prime}} & =C_{\ell}^{b b^{\prime}} \delta_{\ell \ell^{\prime}} \delta_{m m^{\prime}} \\
N_{\ell m, \ell^{\prime} m^{\prime}}^{b, b^{\prime}} & =N^{b} \delta_{b b^{\prime}} \delta_{\ell \ell^{\prime}} \delta_{m m^{\prime}}
\end{aligned}
$$

where $b, b^{\prime}$ label redshift bins, and $\ell, m, \ell^{\prime}, m^{\prime}$ label spherical harmonic modes. The shear angular power spectrum is then given by

$$
C_{\ell}^{b b^{\prime}}=\frac{\pi^{2} \ell}{2} \int_{0}^{\infty} d z \frac{d \chi_{z}}{d z} \frac{W^{b}(z) W^{b^{\prime}}(z)}{\chi_{z}^{3}} \Delta_{\Phi}^{2}(k, z)
$$

where $k=\ell / \chi_{z}$, and $\Delta_{\Phi}^{2}(k, z)$ is the variance of the gravitational potential fluctuations per $\ln k$ interval. The window function is given by

$$
W^{b}(z)=\frac{2 \chi_{z}}{n^{b}} \int_{z_{\min }^{b}}^{z_{\max }^{b}} d z^{\prime} \frac{d n}{d z^{\prime}}\left(1-\frac{\chi_{z}}{\chi_{z^{\prime}}}\right) \Theta\left(z^{\prime}-z\right)
$$

where $\Theta$ is the step function. The diagonal elements of the noise matrix $N$ are $\ell, m$ independent (although they depend on the redshift bin), and given by

$$
N^{b}=\left\langle\gamma_{\text {int }}^{2}\right\rangle_{b} \Omega_{\text {pix }},
$$

where $\left\langle\gamma_{\text {int }}^{2}\right\rangle_{b}$ refers either to $\left\langle\gamma_{1, \text { int }}^{2}\right\rangle$ or to $\left\langle\gamma_{2 \text {,int }}^{2}\right\rangle$, as given by equation (14).

In the late universe, $\Delta_{\Phi}^{2}(k, z)$ is simply related to $\Delta^{2}(k, z)$, the variance of matter density fluctuations per $\ln k$ interval, by

$$
\Delta_{\Phi}^{2}(k, z)=\left(\frac{3 \Omega_{m}}{2 a}\right)^{2}\left(\frac{H_{0}}{c k}\right)^{4} \Delta^{2}(k, z)
$$

where $\Omega_{m}$ is the present-day matter density parameter, $H_{0}$ is the present-day Hubble constant, $a$ is the scale factor normalized to unity today, and $c$ is the speed of light.

This equation neglects fluctuations in dark energy. For a scalar-modeled dark energy, this is assured only for wavelengths shorter than the Compton wavelength of the scalar field, or $k \gg k_{Q}$, where $k_{Q}$ is the Compton wavenumber. Ma et al. [35] gave a fitting formula for $k_{Q}(z)$ when the scalar field equation of state parameter $w$ is a constant and the universe is spatially flat,

$$
k_{Q}=\frac{3 H(a)}{c} \sqrt{(1-w)\left[2+2 w-\frac{w \Omega_{m}}{\Omega_{m}+\left(1-\Omega_{m}\right) a^{-3 w}}\right]} .
$$

Since $k=\ell / \chi$, we require $\ell \gg k_{Q} \chi$ for self-consistency. In a cosmological model close to our fiducial model (defined in the next section) but with $w \rightarrow-1$, the maximum of $k_{Q} \chi$ is $\approx 30$. We follow [15] and impose an upper limit on the angular scales utilized in our study, given by $\ell>40$. Note, however, that dark energy fluctuations are unlikely to be actually detectable on large scales, and our results are insensitive to this lower limit on $\ell$ (see discussion below).

\section{Error Estimates}

We assume the spatial curvature of the universe is zero, and adopt a 7-dimensional cosmological parameter set $\left\{\Omega_{\mathrm{DE}}, \Omega_{m} h^{2}, \sigma_{8}, w_{0}, w_{a}, \Omega_{b} h^{2}, n_{s}\right\}$, where $\Omega_{\mathrm{DE}}, \Omega_{m}, \Omega_{b}$ are present-day energy density parameters of dark energy, total matter (cold dark matter+baryon), and baryons, respectively, $h$ is the Hubble constant in units of $100 \mathrm{~km} \mathrm{~s}^{-1} \mathrm{Mpc}^{-1}, n_{s}$ is the index for the primordial matter power spectrum, and $\sigma_{8}$ is the amplitude of the linear matter density fluctuations today smoothed on a scale of $8 h^{-1} \mathrm{Mpc}$. We consider a time--varying equation of state parameter, given by

$$
w(a)=w_{0}+w_{a}(1-a)
$$

In our fiducial model, we choose the following values of the 7 parameters: $\{0.73,0.14,0.9,-1,0,0.024,1\}$, which are consistent with the current "concordance model" [5].

To estimate uncertainties on the cosmological parameters obtained by a specific probe, we use the Fisher matrix formalism. The Fisher matrix is defined as,

$$
F_{\alpha \beta}=-\left\langle\frac{\partial^{2} \ln L}{\partial p_{\alpha} \partial p_{\beta}}\right\rangle
$$

where $p_{\alpha}, p_{\beta}$ represent the 7 model parameters we want to constrain, $L$ is the likelihood function, the derivatives are evaluated at the true parameter set (which in our case is the adopted fiducial parameter set), and the average is taken over many realizations of the data set. The uncertainty on the parameter $p_{\alpha}$ after marginalized over all other parameters is obtained as $\sqrt{\left(F^{-1}\right)_{\alpha \alpha}}$, which gives a lower limit on the accuracy of $p_{\alpha}$ for any unbiased estimator of the parameters [36], and in the absence of systematic errors.

For number counts, we assume that the counts in each of the 26 redshift bins are independent Poisson random variables, with the mean values given by equation (1). The Fisher matrix in this case is constructed as [37],

$$
F_{\alpha \beta}^{\mathrm{counts}}=\sum_{i=1}^{26} \frac{\partial \bar{N}_{i}}{\partial p_{\alpha}} \frac{\partial \bar{N}_{i}}{\partial p_{\beta}} \frac{1}{\bar{N}_{i}}
$$

For the shear-shear correlations, we assume that the spherical harmonic expansion coefficients are Gaussian random variables whose mean are zero, and whose covariance matrix is $\mathbf{C}^{\gamma \gamma}$. The Fisher matrix is then constructed as 38]

$$
F_{\alpha \beta}^{\gamma \gamma}=\frac{1}{2} f_{\text {sky }} \sum_{\substack{\ell, b_{1}, b_{2}, b_{3}, b_{4}}}(2 \ell+1) C_{\ell, \alpha}^{b_{1} b_{2}} W_{\ell}^{b_{2} b_{3}} C_{\ell, \beta}^{b_{3} b_{4}} W_{\ell}^{b_{4} b_{1}}
$$


where $f_{\text {sky }}$ is the fraction of the sky coved by the survey (in our case, $f_{\text {sky }}=0.44$ ), $[X]_{, \alpha}$ denotes the derivative of $[X]$ with respect to $p_{\alpha}, C_{\ell}^{b b^{\prime}}$ is calculated from equation (18), and the $W_{\ell}^{b b^{\prime}}$ are the elements of the inverse of the covariance matrix,

$$
\begin{aligned}
\mathbf{W} & =\left(\mathbf{C}^{\gamma \gamma}\right)^{-1} \\
W_{\ell m, \ell^{\prime} m^{\prime}}^{b, b^{\prime}} & =W_{\ell}^{b b^{\prime}} \delta_{\ell \ell^{\prime}} \delta_{m m^{\prime}} .
\end{aligned}
$$

Non-linear gravitational clustering in the late universe will induce non-Gaussian signatures in matter fluctuation field. Although we incorporate these nonlinear effects on the matter power spectrum, these nonlinear effects will also render the likelihood function nonGaussian. To avoid this complication, we neglect all modes with $\ell>1000$ [39].

To compute the linear matter power spectrum, we use KINKFAST [40], a version of CMBFAST [41] modified to accommodate a time-varying equation of state parameter $w$, to calculate the transfer function at $z=0$, and we obtain the linear growth function by integrating the differential equations given in the Appendix of ref. 22]. For the shear-shear correlations, the non-linear matter power spectrum is constructed following Smith et al. [42]. The derivatives in the Fisher matrices are calculated by two-sided numerical approximations. For both $F^{\text {counts }}$ and $F^{\gamma \gamma}$, we chose a step-size of $\Delta w_{a}= \pm 0.01$, and $\pm 1 \%$ of the fiducial value for the other 6 parameters. We have verified directly that these step-sizes are small enough for the Fisher matrix entries to have converged.

\section{Covariance Between Number Counts and Shear}

Our treatments of the number counts (only shot noise is considered) and its combination with shear-shear correlations (covariance between these two are neglected) are quite simplified. To yield more accurate predictions, sample variance errors for the number counts, and the covariance between number counts and shear-shear correlations should be taken into account.

The effect of sample variance on the constraining power of number counts has been considered in detail in previous work [43, 44]. In particular, ref. [43] finds (see their Figure 2) that the degradation on dark energy constraints, when the sample variance error is added to the Poisson error, depends mostly on the mass threshold. At the lower end of the range of our fiducial mass thresholds, $\sim(0.6-4) \times 10^{14} \mathrm{M}_{\odot}$, the degradation is a factor of $\sim 2$, while at the upper end, there is negligible degradation (see their Figure 7). However, these degradations are overestimates, because ref. [43] excluded the "signal" that arises from the cosmology dependence of the sample variance. When this information is included, sample variance errors should cause a smaller degradation in the number count constraints (and possibly even improvement, if the survey is sub-divided into many angular cells, as in [44]).
For our purposes, the more important question is whether the covariance between number counts and shear-shear correlations is significant. The potential concern is that the shear-shear correlations probe the same realization of the density field as the cluster counts, and therefore simply adding the constraints from the two observables may overestimate their combined constraining power. Indeed, in the hypothetical limit that the mean cluster abundance in a particular direction and redshift bin is fully predictable, given measurements of the shear correlations, the cluster counts should not yield any new information on dark energy. However, we show here that the covariance is very small, and the two observables can safely be regarded as independent. In this section, we summarize the results of the covariance calculation; the interested reader is encouraged to consult the Appendix for details.

In the general case, the Fisher matrix, defined in equation (24), involves the expectation value of the derivatives of the joint likelihood function $L=L(\mathbf{x}, \mathbf{p})$ where $\mathbf{x}$ is a vector of the observables, and $\mathbf{p}$ is a vector of the model parameters. In our case, $\mathbf{x}$ contains the number counts in 26 redshift bins, $\left\{N_{i} ; 1 \leq i \leq 26\right\}$, and the spherical expansion coefficients in 8 different redshift bins $\left\{a_{\ell m}^{b} ; 1 \leq B \leq 8,41 \leq \ell \leq 1000,-(\ell+1) \leq m_{\ell} \leq(\ell+1)\right\}$ (note that we have $8,002,560+26=8,002,586$ observables). Here both $N_{i}$ and $a_{\ell m}^{b}$ are random variables, and in the discussion below, the probability distribution of both are taken to be determined by large-scale density fluctuations alone. In practice, the measurement of either quantity represents a discrete sampling of a continuous random field, and will therefore have an additional sampling error. In particular, equation (25) assumes that Poisson errors dominate the sample variance errors, whereas equation (26) incorporates the additional stochastic noise from the distribution if intrinsic shapes. However, such sampling errors should be uncorrelated and will be ignored below. Note that excluding truly uncorrelated errors is conservative, since they would reduce the cross-correlation coefficient defined below (eq 30 ).

Under the assumption that the full joint likelihood function is Gaussian, the Fisher matrix depends only on the mean $\overline{\mathbf{x}}$ and the covariance matrix $\mathbf{C}=\langle(\mathbf{x}-$ $\left.\overline{\mathbf{x}})(\mathbf{x}-\overline{\mathbf{x}})^{T}\right\rangle$, and on the derivatives of these quantities with respect to the model parameters $\mathbf{p}$ [36] (note that in general, $\overline{\mathbf{x}}$ and $\mathbf{C}$ both depend on $\mathbf{p}$ ). In our case, the full covariance matrix $\mathbf{C}$ contains the terms $\left\langle\left(N_{i}-\bar{N}_{i}\right)\left(N_{j}-\bar{N}_{j}\right)\right\rangle$ and $\left\langle a_{\ell m}^{b} a_{\ell^{\prime} m^{\prime}}^{b^{\prime}{ }^{\prime}}\right\rangle$, which describe the sample variance in the number counts and in the shear field, respectively. ${ }^{1}$ The cross-terms, $\left\langle\left(N_{i}-\bar{N}_{i}\right) a_{\ell m}^{b}\right\rangle$, describe the covariance between number counts and the shear field. Here $\bar{N}_{i}$ is the mean number of clusters given

\footnotetext{
${ }^{1}$ Note that eq. (26) depends on $C_{\ell}$ and its derivatives, rather than $a_{\ell m}$. This dependence arises from taking the expectation value $C_{\ell} \equiv[1 /(2 \ell+1)]\left\langle\Sigma_{m=-\ell}^{+\ell}\left|a_{\ell m}\right|^{2}\right\rangle$.
} 




FIG. 1: Absolute values of the cross-correlation coefficients (defined in eq30 in the text) between the number of galaxy clusters in redshift range of $(0.1<z<0.15)$ and the spherical harmonic expansion coefficients (at $m=0$ ) of the shear map from source galaxies in redshift range of $(0<z<0.4)$. The figure shows that the cross-correlation coefficients are of order $10^{-3}$ or smaller for $\ell>40$.

in equation (11), while we have $\overline{a_{\ell m}^{b}}=0$, and the averages are taken over many realization, or a large volume.

In the Appendix, we calculate the cross-terms explicitly, and show that they are given by a simple expression,

$$
\begin{array}{r}
\left\langle\left(N_{i}-\bar{N}_{i}\right) a_{\ell m}^{b}\right\rangle=\delta_{m 0} \frac{3 \pi^{2}}{2} \frac{\Omega_{m} H_{0}^{2}}{c^{2}} \frac{\chi_{z_{i}}\left(1+z_{i}\right)}{\ell^{3}} \times \\
\tilde{\Theta}_{\ell} N_{i} b\left(z_{i}\right) W^{b}\left(z_{i}\right) \Delta^{2}\left(k=\frac{\ell}{\chi_{z_{i}}}, z_{i}\right) .
\end{array}
$$

Here $\tilde{\Theta}_{\ell}$ is the spherical harmonic transform of an azimuthally symmetric angular window function, $W^{b}$ is the lensing window function (given in equation 19), and $b(z)$ is the mean bias factor of the cluster counts (averaged over clusters above the detection threshold). The above result assumes that the cluster number counts trace the matter density field with the linear bias factor $b$, and we have also used the Limber approximation. The latter assumption should be justified for the angular modes we use $(\ell>40)$. Note that the cross-term vanishes for clusters behind the source galaxies (since $W^{b}\left(z_{i}\right)=0$ for $z_{i}>z_{\max }^{b}$ ), and also for $m \neq 0$ (since our survey window is azimuthally symmetric).

We define the cross-correlation coefficient between the $N_{i}$ and the $a_{\ell m}^{b}$ as

$$
\xi_{i, b \ell m} \equiv \frac{\left\langle\left(N_{i}-\bar{N}_{i}\right) a_{\ell m}^{b}\right\rangle}{\sqrt{\left\langle\left(N_{i}-\bar{N}_{i}\right)^{2}\right\rangle\left\langle\left|a_{\ell m}^{b}\right|^{2}\right\rangle}} .
$$

As an example, here we calculate the cross-correlation coefficient for the number counts and the shear field in their lowest respective redshift bins, $\left|\xi_{1,1 \ell 0}\right|$. The results

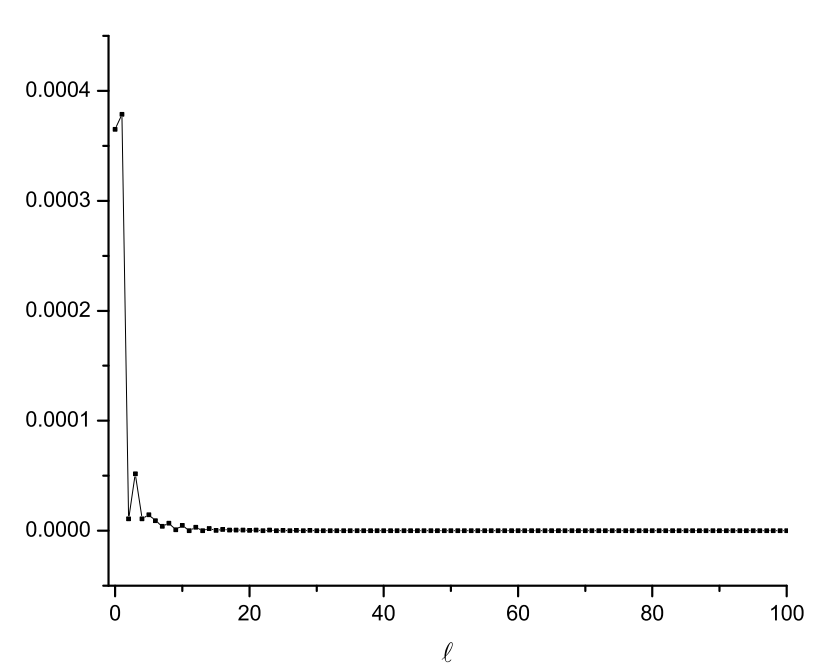

FIG. 2: Contributions from different $\ell$-modes to the sample variance for the number of galaxy clusters in the first redshift bin $(0.1<z<0.15)$, containing, on average, 7515 clusters. The figure shows that the sample variance is dominated by the largest angular scales. This explains the lack of crosscorrelation between the counts and the shear field, when the latter is restricted to smaller angular scales $(\ell>40)$.

are shown, as a function of $\ell$, in Figure 11. The figure shows that the $\left|\xi_{1,1 \ell 0}\right|$ are small - of order $10^{-3}$ or less - for $\ell>40$. For a different pair of redshifts, $i$ and $B$, we expect this order of magnitude would not change significantly, since the related quantities vary slowly with redshift.

The fact that the $\xi_{i, b \ell m}$ are small can be explained by the following reasoning. For a given survey window, the variance in the cluster counts alone is dominated by the largest angular modes, due to cancellations among smaller-scale fluctuations along the direction transverse to the line of sight. In Figure 2, we explicitly show the contributions to the sample variance of the cluster counts in the first redshift bin for LSST (note that we do not use the Limber approximation for this calculation). Because of the large angular size of the window, the figure clearly shows that the variance is small for modes with $\ell \gtrsim 10$. Fluctuations in the underlying (isotropic) matter density field on different scales $\ell$ are uncorrelated, and we have limited the range of angular modes of the shear maps to $\ell>40$. Since these relatively smaller-scale modes contribute little to the fluctuations in the number counts, we indeed expect the $\xi_{i, b \ell m}$ to be small.

According to these results, the probability of drawing a set of $N_{i}$ and $a_{\ell, m}^{b}$ is given by a multi-variate Gaussian, with the total covariance matrix that consists of four blocks,

$$
\mathbf{C}_{\text {tot }}=\left(\begin{array}{cc}
\mathbf{S}^{\text {counts }} & \mathbf{C}^{\text {cross }} \\
\left(\mathbf{C}^{\text {cross }}\right)^{\mathbf{T}} & \mathbf{S}^{\gamma \gamma}
\end{array}\right)
$$


where $\mathbf{S}^{\text {counts }}$ and $\mathbf{S}^{\gamma \gamma}$ are the sample variance matrices for counts and shear alone. When $\left|\xi_{i, b \ell m}\right| \ll 1$, this matrix can be well approximated (for example, for the purpose of taking its inverse) by

$$
\mathbf{C}_{\text {tot }}=\left(\begin{array}{cc}
\mathbf{S}^{\text {counts }} & \mathbf{0} \\
\mathbf{0} & \mathbf{S}^{\gamma \gamma}
\end{array}\right) \text {. }
$$

A full treatment would incorporate the Poisson errors for the counts, and the shape-errors for the shear, however, these effects are relatively small, and could only decrease the value of $\xi$. The Fisher matrix also requires taking the derivatives with respect to the cosmological parameters. As long as these derivatives do not have a strong scale-dependence, and given the smallness of $\xi$, we expect our basic conclusion to carry over to the Fisher matrix. Therefore, the cluster number counts and shearshear correlations can be treated as independent probes, even if they probe the same area of the sky. For the results below, we therefore simply add the two Fisher matrices, when we combine the two observables.

\section{RESULTS}

The marginalized errors on the seven cosmological parameters from the number counts and the shear-shear correlations are listed in the $2^{\text {nd }}$ and $3^{\text {rd }}$ columns of Table I for our fiducial LSST-like survey. Note that the results scale simply as $\Delta \Omega^{-1 / 2}$ for a survey with a different solid angle coverage. The $4^{\text {th }}$ column shows the result from combining the two observable, assuming that they are independent, so that the two Fisher matrices can simply be added (see the discussion of the covariance between these two probes in $\S \llbracket D$ above). In the limit that the two observable have the same degeneracies between parameters, their combination would be equivalent to simply adding the marginalized errors in quadrature. In the $5^{\text {th }}$ column of Table I] we show a "complementarity" parameter, $\eta$, which quantifies the effect of degeneracy-breaking that occurs when the two methods are combined. The parameter $\eta$ is defined as the improvement of the constraints beyond adding the two results in quadrature,

$$
\eta=\left(\Delta p_{\alpha}^{\text {counts }+\gamma \gamma}\right)^{2}\left[\frac{1}{\left(\Delta p_{\alpha}^{\text {counts }}\right)^{2}}+\frac{1}{\left(\Delta p_{\alpha}^{\gamma \gamma}\right)^{2}}\right] .
$$

With this definition, $\eta=1$ corresponds to no degeneracy breaking, and lower values of $\eta$ indicate larger benefits from the combination. The $6^{\text {th }}$ and $7^{\text {th }}$ columns are the same as the $3^{\text {rd }}$ and $4^{\text {th }}$, except that, as an academic exercise, for the shear-shear correlations, we use the linear matter power spectrum instead of the nonlinear one (see the next section for a detailed discussion).

The first conclusion to draw from Table \ is that the shear-shear correlations give tighter constraints on all cosmological parameters than cluster counts alone, especially on $\Omega_{\Lambda}, \Omega_{m} h^{2}, \sigma_{8}$ and $w_{a}$. This may not be surprising, given that number counts effectively measure only 26
TABLE I: This table contains our main results. Marginalized errors are shown on cosmological parameters from cluster counts, shear-shear correlations, and their combination. The parameter $\eta$, shown in the $5^{\text {th }}$ column, measures the synergy between the two observable, with $\eta=1$ indicating no synergy, and lower values indicating significant degeneracy-breaking (see text for definition). In the $6^{\text {th }}$ and $7^{\text {th }}$ columns, we use the linear power spectrum for the shear (indicated here, and in the other tables below, by the superscript "l"). Priors from WMAP, $\Delta \Omega_{b} h^{2}=0.0010, \Delta n_{s}=0.040$, are included for the $2^{\text {nd }}, 4^{\text {th }}$, and $7^{\text {th }}$ columns here, and in columns involving number counts in all other tables below (except Table IV).

\begin{tabular}{|c|c|c|c|c|c|c|}
\hline & counts & $\gamma \gamma$ & counts $+\gamma \gamma$ & $\eta$ & $\gamma \gamma^{l}$ & counts $+\gamma \gamma^{l}$ \\
\hline$\Delta \Omega_{\mathrm{DE}}$ & 0.064 & 0.0084 & 0.0026 & 0.097 & 0.013 & 0.0027 \\
$\Delta \Omega_{m} h^{2}$ & 0.20 & 0.049 & 0.0061 & 0.017 & 0.034 & 0.0050 \\
$\Delta \sigma_{8}$ & 0.029 & 0.012 & 0.0031 & 0.083 & 0.021 & 0.0031 \\
$\Delta w_{0}$ & 0.080 & 0.078 & 0.033 & 0.34 & 0.12 & 0.031 \\
$\Delta w_{a}$ & 1.24 & 0.28 & 0.11 & 0.18 & 0.42 & 0.099 \\
$\Delta \Omega_{b} h^{2}$ & 0.0010 & 0.014 & 0.00099 & 0.99 & 0.010 & 0.00099 \\
$\Delta n_{s}$ & 0.040 & 0.050 & 0.016 & 0.26 & 0.027 & 0.011 \\
\hline
\end{tabular}

numbers, while the shear power spectrum is effectively a measurement of many more parameters. On the other hand, cluster counts deliver a constraint comparable to that from the shear for $w_{0}$. We also note that constraints from cluster counts alone on $w_{a}$ are weak (as noted by [20], this can be significantly improved by adding the cluster power spectrum and CMB anisotropy as observable). ${ }^{2}$

More importantly, Table I shows that when the two methods are combined, the constraints on the cosmological parameters improve significantly. For most of the parameters, $\eta$ is small, indicating significant complementarity. In particular, focusing on the three dark-energy parameters, the combination tightens the constraints by a factor of 3-10 more than simply adding the marginalized errors in quadrature. The combined constraint on $\Omega_{\mathrm{DE}}$ is $\sim 25$ times better than that from counts and $\sim 3$ times better than that from $\gamma \gamma$; the combined constraint on $w_{0}$ is $\sim 2$ times better than either from counts or $\gamma \gamma$; and the combined constraint on $w_{a}$ is $\sim 11$ times better than from counts and $\sim 3$ times better than from $\gamma \gamma$. These results are also shown graphically in Figures 3 and 4.

For reference, we follow the recommendation of the Dark Energy Task Force (DETF) [45], and compute the

\footnotetext{
${ }^{2}$ We note for reference that our shear correlation constraints are consistent with a slightly updated version of the results in ref. [15]. Our number-count-alone constraints on $\Omega_{\mathrm{DE}}$ and $w_{a}$, on the other hand, are significantly weaker than in ref. [20]. We have found that the discrepancy is due to inaccurate interpolation in ref. 20] to obtain the mass limit. We have also found, however, that the inaccuracies do not significantly alter the joint constraints when the number counts are combined with other observables.
} 


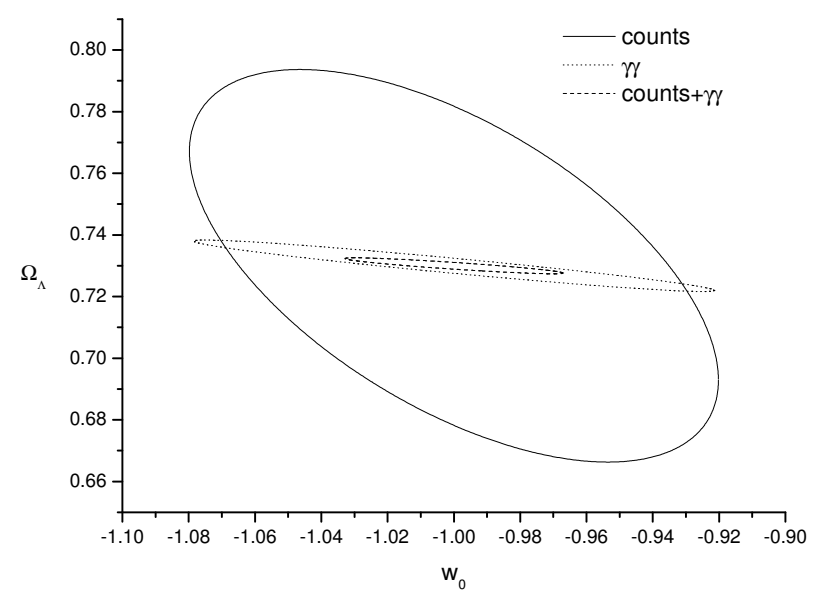

FIG. 3: Marginalized constraints in the $\left(\Omega_{\mathrm{DE}}, w_{0}\right)$ plane for an LSST-like survey from the shear-selected cluster counts, the shear-shear correlations, and the combination of these two observables. Note that the cluster counts alone deliver weaker constraints, but still improve the $w_{0}$ errors from the shear-shear correlations by a factor of $\sim$ two, as a result of breaking degeneracies.

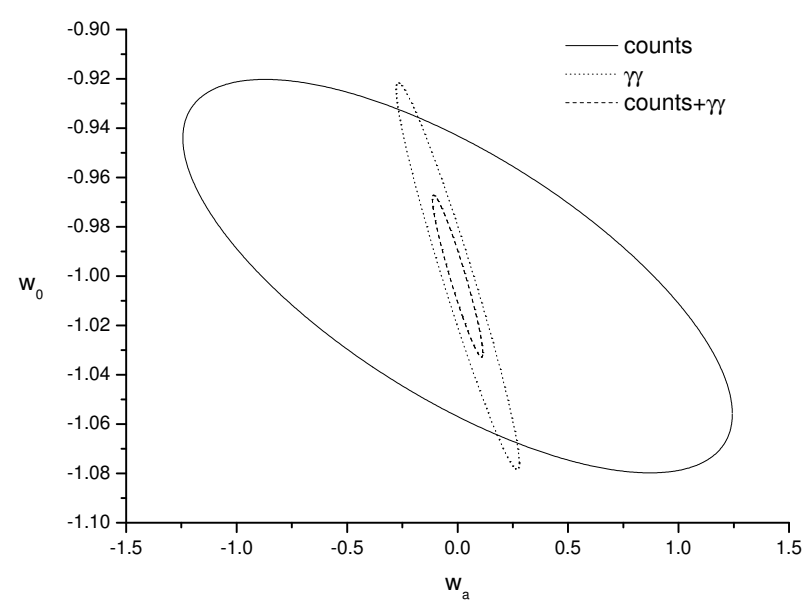

FIG. 4: Marginalized constraints in the $\left(w_{0}, w_{a}\right)$ plane for an LSST-like survey from the shear-selected cluster counts, the shear-shear correlations, and the combination of these two observables.

"pivot point" $-a_{p}$, i.e. the value of the scale factor where $w(a)$ is best constrained. In Table III, we list the values of the scale factor and redshift of this pivot point, as well as the errors on $w\left(a_{p}\right)$ and the "figure of merit" defined by the DETF, $\left(\Delta w_{p} \Delta w_{a}\right)^{-1}$. The figure of merit we find for the individual observable is in-between the "optimistic" and "pessimistic" predictions by the DETF. The combined figure of merit, however, is significantly better than the most optimistic figure of merit in the
TABLE II: This table recast our results in terms of a pivot point. The pivot point is defined as the scale factor at which the equation of state $w_{p} \equiv w\left(a_{p}\right)$ is best constrained. The last row shows the figure-of-merit proposed by the DETF [45].

\begin{tabular}{|c|c|c|c|}
\hline & counts & $\gamma \gamma$ & counts $+\gamma \gamma$ \\
\hline$z_{p}$ & 0.047 & 0.37 & 0.37 \\
$1-a_{p}$ & 0.045 & 0.27 & 0.27 \\
$\Delta w_{p}$ & 0.057 & 0.020 & 0.010 \\
$\Delta w_{a}$ & 1.24 & 0.28 & 0.11 \\
$\left(\Delta w_{p} \Delta w_{a}\right)^{-1}$ & 14.1 & 178.6 & 909.1 \\
\hline
\end{tabular}

DETF for a "stage II" WL shear experiment alone.

\section{DISCUSSION}

In this section, we discuss several aspects of our basic simple results presented in the previous section. In particular, we explain the reasons for the synergy between the two observable, and discuss various possible caveats that could modify our conclusions.

\section{A. Degeneracy Breaking by the Two Observables}

Table III lists the uncertainties on the 7 cosmological parameters when fixing all the other 6 parameters at their fiducial value. By comparing Table【with Table【. we see that, using either observable alone, degeneracies among the 7 parameters lead to great degradation on the constraints. Clearly, combining these two observables can decrease the effect of this degradation by breaking degeneracies. To understand this better, we find the worstconstrained directions in the 7D parameter space for both of the observables. We do this by diagonalizing the Fisher matrix, and finding the eigenvector that corresponds to the smallest eigenvalue, i.e. whose direction is the one along which the probe is least sensitive at and hence constrained worst. For the number counts, we find that the $\left(\Omega_{\mathrm{DE}}, \Omega_{m} h^{2}, \sigma_{8}, w_{0}, w_{a}, \Omega_{b} h^{2}, n_{s}\right)$-components of the unit vector pointing in this direction are $(0.050,0.15$, $0.023,-0.044,0.99,0,-0.00016)$, with the corresponding eigenvalue of 0.63 . This implies that constraints on $\left(\Omega_{\mathrm{DE}}, w_{0}, w_{a}\right)$ can not be better than $(0.063,0.056,1.24)$. For shear-shear correlations, the direction of the worst degeneracy is $(0.026,0.084,0.038,-0.26,0.95,0.022$, 0.10 ), with a corresponding eigenvalue of 12 . Again, this implies that constraints on $\left(\Omega_{\mathrm{DE}}, w_{0}, w_{a}\right)$ can not be better than $(0.0077,0.076,0.28)$. In both cases, we find that the $2^{\text {nd }}$ worst eigenvalue is much greater that the worst by a factor of $\sim 5^{2}$ for the shear-shear correlations, and by a factor of $17^{2}$ for the number counts. Apparently the 7-dimensional error ellipsoid is very narrow, with a large extension in one direction that nearly (but not exactly) coincides with the $w_{a}$ axis. 
TABLE III: This table shows the effect of marginalization. The parameter errors are shown from the two observables, as in Table [ but before marginalization (i.e. assuming the other 6 parameters are fixed).

\begin{tabular}{|c|c|c|}
\hline & counts & $\gamma \gamma$ \\
\hline$\Delta \Omega_{\mathrm{DE}}$ & 0.00036 & 0.00041 \\
$\Delta \Omega_{m} h^{2}$ & 0.0013 & 0.0015 \\
$\Delta \sigma_{8}$ & 0.00042 & 0.00064 \\
$\Delta w_{0}$ & 0.0054 & 0.0060 \\
$\Delta w_{a}$ & 0.029 & 0.024 \\
$\Delta \Omega_{b} h^{2}$ & 0.00050 & 0.00060 \\
$\Delta n_{s}$ & 0.0034 & 0.0044 \\
\hline
\end{tabular}

TABLE IV: This table shows the effect of the WMAP priors. Marginalized errors are shown from cluster counts, and the combination of the counts and the shear-shear correlations, as in the $2^{\text {nd }}$ and $3^{\text {rd }}$ columns of Table I. except that we exclude the WMAP priors.

\begin{tabular}{|c|c|c|}
\hline & counts $^{\text {nopriors }}$ & $(\text { counts }+\gamma \gamma)^{\text {nopriors }}$ \\
\hline$\Delta \Omega_{\mathrm{DE}}$ & 0.15 & 0.0027 \\
$\Delta \Omega_{m} h^{2}$ & 3.92 & 0.045 \\
$\Delta \sigma_{8}$ & 0.067 & 0.0034 \\
$\Delta w_{0}$ & 0.20 & 0.037 \\
$\Delta w_{a}$ & 1.40 & 0.14 \\
$\Delta \Omega_{b} h^{2}$ & 1.46 & 0.013 \\
$\Delta n_{s}$ & 5.04 & 0.044 \\
\hline
\end{tabular}

Given the very severe degeneracies, and the fact that they do not point in the same direction for the two observables, it is not surprising that the combination of the two observables leads to a tightening of the constraints beyond adding the marginalized errors in quadrature.

Finally, since we have added WMAP priors for the number counts constraints, it is useful to ask how important these priors were for the combined errors. In Table IV] we show the constraints from the cluster counts, and the combination of the counts and the shear-shear

TABLE V: This table shows the effect of including shear measurements on small angular scales. Marginalized errors are shown on the cosmological parameters, as in Table I] The difference from Table【 is that we have used additional smallscales for the shear-shear correlations, by increasing the cutoff from $\ell_{\max }=1000$ to $\ell_{\max }=3000$.

\begin{tabular}{|c|c|c|c|c|c|c|}
\hline & counts & $\gamma \gamma$ & counts $+\gamma \gamma$ & $\eta$ & $\gamma \gamma^{l}$ & counts $+\gamma \gamma^{l}$ \\
\hline$\Delta \Omega_{\mathrm{DE}}$ & 0.064 & 0.0046 & 0.0023 & 0.25 & 0.012 & 0.0025 \\
$\Delta \Omega_{m} h^{2}$ & 0.20 & 0.034 & 0.0050 & 0.023 & 0.021 & 0.0039 \\
$\Delta \sigma_{8}$ & 0.029 & 0.0060 & 0.0027 & 0.21 & 0.019 & 0.0029 \\
$\Delta w_{0}$ & 0.080 & 0.046 & 0.025 & 0.38 & 0.11 & 0.028 \\
$\Delta w_{a}$ & 1.24 & 0.15 & 0.079 & 0.27 & 0.39 & 0.084 \\
$\Delta \Omega_{b} h^{2}$ & 0.0010 & 0.010 & 0.00099 & 1.0 & 0.0072 & 0.00099 \\
$\Delta n_{s}$ & 0.040 & 0.026 & 0.0097 & 0.19 & 0.011 & 0.0054 \\
\hline
\end{tabular}

correlations, as in the $2^{\text {nd }}$ and $3^{\text {rd }}$ columns of Table I, except that we exclude the WMAP priors. As Table IV shows, the clusters counts are insensitive to $\Omega_{b} h^{2}$ and $n_{s}$, and degeneracies with these parameters also degrade the constraints on other parameters. However, the shearshear correlation provides a sufficiently accurate measurement of $\Omega_{b} h^{2}$ and $n_{s}$, and the WMAP priors are not important for the combined constraints.

\section{B. Information from Small-Scale Shear-Shear Correlations and Non-Linearities}

In the above results, we have imposed a small-scale cutoff, $\ell_{\max }=1000$ for the shear-shear correlations. It is possible, however, at least in principle, to obtain accurate non-linear shear power spectra, using numerical simulations on a large grid of cosmological parameters. It is therefore interesting to ask whether including higher $\ell$ modes could improve the final results significantly. To answer this question, we repeated all the calculations in Table I but this time with $\ell_{\max }=3000$. The results are listed in Table $\mathrm{V}$

First, we notice from either Table 1 or Table $\mathrm{V}$, that constraints from the shear-shear correlations alone are better when the non-linear power spectrum is used than those from the linear version. This suggests that there is extra information in the shear-shear correlations that comes from the non-linear effects. Furthermore, compar-

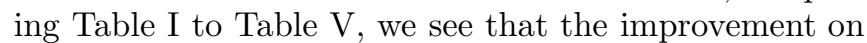
dark energy constraints from these non-linearities is only about $50 \%$ for $\ell_{\max }=1000$, but increases to a factor of $2-3$ for $\ell_{\max }=3000$. However, Tables $\square$ and $\nabla$ both show that once the cluster count information is added, the nonlinear effects on the shear-shear power spectrum become essentially irrelevant, even for $\ell_{\max }=3000$.

This indicates that while non-linear effects change the shear-shear power spectrum, the information content of these changes is sub-dominant compared with that probed by number counts, at least for the non-linearities contained in modes with $\ell \leq 3000$ (note that clusters are strongly nonlinear objects). Given these results, we are satisfied to neglect the higher $\ell$ modes and stick to our original choice of $\ell \leq 1000$. The fact that higher $\ell$ modes $(3000 \geq \ell>1000)$ help little $(\sim 10 \%)$ on the linear shear-shear correlations, but help more $(\sim 50 \%)$ with the nonlinear shear-shear correlations, together with the fact that adding higher $\ell$ modes help little (30\% at most) in either case when the number counts are included, is again an indication that the nonlinear evolution information in these modes is sub-dominant compared to the information contained in number counts. 
TABLE VI: This table shows the effect of excluding shear measurements on the largest scales. Marginalized errors on the cosmological parameters, as in Table [ The difference from Table \ is that we have neglected large-scales for the shearshear correlations, by increasing the cutoff from $\ell_{\text {min }}=41$ to $\ell_{\min }=100$. As the comparison of the two tables show, the constraints do not degrade significantly, implying that most of the information is at relatively small angular scales $(\ell>100)$.

\begin{tabular}{|c|c|c|c|c|c|c|}
\hline & counts & $\gamma \gamma$ & counts $+\gamma \gamma$ & $\eta$ & $\gamma \gamma^{l}$ & counts $+\gamma \gamma^{l}$ \\
\hline$\Delta \Omega_{\mathrm{DE}}$ & 0.064 & 0.0087 & 0.0026 & 0.094 & 0.014 & 0.0027 \\
$\Delta \Omega_{m} h^{2}$ & 0.20 & 0.051 & 0.0075 & 0.023 & 0.036 & 0.0060 \\
$\Delta \sigma_{8}$ & 0.029 & 0.012 & 0.0032 & 0.081 & 0.022 & 0.0031 \\
$\Delta w_{0}$ & 0.080 & 0.081 & 0.034 & 0.35 & 0.13 & 0.032 \\
$\Delta w_{a}$ & 1.24 & 0.29 & 0.12 & 0.18 & 0.45 & 0.10 \\
$\Delta \Omega_{b} h^{2}$ & 0.0010 & 0.015 & 0.0010 & 1.0 & 0.012 & 0.00099 \\
$\Delta n_{s}$ & 0.040 & 0.052 & 0.019 & 0.38 & 0.028 & 0.013 \\
\hline
\end{tabular}

\section{Large Scale Shear-Shear Correlations and Dark Energy Clustering}

The previous sub-section showed that while most of the information in the non-linear shear-shear correlations is on small scales, the information contained in the linear shear-shear correlations is coming mostly from larger scales $\ell<1000$. For completeness, we here ask whether the largest scales $(\ell \sim 40)$ actually dominate shear-shear information. We show, in TableVI, the constraints, recalculated as in Table I. except we have neglected large-scales for the shear-shear correlations, by increasing the cutoff from $\ell_{\min }=41$ to $\ell_{\min }=100$. As the comparison of the two tables show, the constraints do not degrade significantly, implying that most of the information is at smaller angular scales $(\ell>100)$.

A related issue is that in scalar field models of dark energy (e.g. 28]), such as quintessence [46], the field clusters on large scales, while it remains smooth on small scales. This is different from a cosmological constant, which remains smooth on all scales, and the additional dark-energy fluctuations can enhance the matter fluctuations on large scales. It is interesting to ask whether this enhancement may be detectable through the shear-shear correlations [11]. We take, as an example, the shearshear auto power spectra $\left(C_{\ell}^{88}\right)$ of the 8th. Compared with other bins, this has the largest comoving radial distance, so that for fixed $\ell$, it probes the largest comoving scales, and should be most sensitive to the clustering effect of quintessence field. We calculate $C_{\ell}^{88}$ for both our fiducial model (with a cosmological constant) and a quintessence cosmological model with $w_{0}=-0.5$, with all other parameters fixed. In the quintessence model, we use KINKFAST with the choice for the transfer function that includes dark energy perturbations.

The quintessence field affects the power spectrum through the expansion rate, the growth rate and the enhancement of the matter fluctuations on large scales, causing the deviations of these two $C_{\ell}^{88}$ curves from each other. To separate the clustering effect from the other two effects, we artificially replace the transfer function in the $w=-0.5$ quintessence cosmological model by the one that excludes the effect of dark energy clustering (i.e. the $w=-1$ transfer function), and calculate $C_{\ell}^{88}$ for the quintessence cosmological model again. We find that the difference caused by dark energy clustering is unfortunately quite small, safely within the error bars. A remaining issue is that the above treatment only computes the fluctuations in the matter density induced by dark energy clustering. On the other hand, the weak lensing signal is sensitive to fluctuations in the total gravitational potential, which has an additional contribution directly from the fluctuations in the dark energy component. However, we expect these two contributions to be of similar order of magnitude. We conclude, in agreement with ref. [11] that while the shear-shear correlations can tell the quintessence field apart from a cosmological constant, the distinction is made purely through the effect on the growth rate and expansion rate, and the clustering of dark energy on large scales remains undetectable.

\section{Shear Power Spectrum vs. Cluster Power Spectrum}

Once the galaxy clusters are detected, their spatial distribution, characterized, e.g., by the cluster power spectrum $\left(P_{c}(k)\right)$, readily offers another constraint on cosmology. Since earlier works [20, 47] have studied the complementarity of the cluster counts and their power spectrum, here we contrast the $d N / d z+P_{c}(k)$ combination with the $d N / d z+C_{\ell}$ combination. To perform this comparison, we divide the clusters according to their redshift into 6 bins, each with size of $\Delta z=0.2$, except the farthest one with size of $\Delta z=0.3$. For each bin, we follow $\mathrm{Hu} \&$ Haiman [48] and compute the cluster power spectrum over $30 \times 30 k$-space cells centered at $k_{\|}, k_{\perp}=0.005,0.010, \ldots, 0.15 \mathrm{Mpc}^{-1}$, where $k_{\|}, k_{\perp}$ are wave-numbers parallel and transverse to the line of sight, respectively. The methods to obtain constraints on cosmological parameters from the cluster power spectrum are the same as those described in [20]. Our results are shown in Table VII separately for the number counts (as before), the power spectrum, and their combination. We have simply summed the two Fisher matrices, assuming that the two measurements are independent and have no covariance. This assumption was implicitly made in previous works [20, 47], but it could be justified by arguments similar to those in the previous section. The $5^{\text {th }}$ column in the Table shows the complementarity parameter, defined analogously to that in Table [1

A comparison of Table [I and Table VII reveals that shear-shear correlations give tighter constraints on $\Omega_{\mathrm{DE}}, w_{0}, w_{a}$ than the cluster power spectrum, either by itself, or in combination with the number counts. In particular, the combined constraints are a factor of $\sim 1.5-2$ better when the shear-shear correlations are 
TABLE VII: This table replaces the shear-shear correlations by the cluster power spectrum. Marginalized errors are shown on cosmological parameters from cluster counts, cluster power spectrum, and their combination. The parameter $\eta$, shown in the $5^{\text {th }}$ column, measures the synergy between the two observables, as in Table 1.

\begin{tabular}{|c|c|c|c|c|}
\hline & counts & $P_{c}(k)$ & counts $+P_{c}(k)$ & $\eta$ \\
\hline$\Delta \Omega_{\mathrm{DE}}$ & 0.064 & 0.0095 & 0.0040 & 0.18 \\
$\Delta \Omega_{m} h^{2}$ & 0.20 & 0.027 & 0.0047 & 0.030 \\
$\Delta \sigma_{8}$ & 0.029 & 0.020 & 0.0050 & 0.091 \\
$\Delta w_{0}$ & 0.080 & 0.12 & 0.057 & 0.75 \\
$\Delta w_{a}$ & 1.24 & 0.54 & 0.23 & 0.23 \\
$\Delta \Omega_{b} h^{2}$ & 0.0010 & 0.0063 & 0.00097 & 0.97 \\
$\Delta n_{s}$ & 0.040 & 0.046 & 0.013 & 0.19 \\
\hline
\end{tabular}

TABLE VIII: This table shows the effect of excluding the highest redshift galaxies from the shear measurements. Marginalized errors are shown on the cosmological parameters, as in Table [ except that here we have used only the first four redshift bins for the shear-shear correlations, that is, only the source galaxies with redshift $[0,1.6]$ are considered.

\begin{tabular}{|c|c|c|c|c|c|c|}
\hline & counts & $\gamma \gamma$ & counts $+\gamma \gamma$ & $\eta$ & $\gamma \gamma^{l}$ & counts $+\gamma \gamma^{l}$ \\
\hline$\Delta \Omega_{\mathrm{DE}}$ & 0.064 & 0.011 & 0.0034 & 0.11 & 0.035 & 0.0035 \\
$\Delta \Omega_{m} h^{2}$ & 0.20 & 0.11 & 0.0095 & 0.0093 & 0.065 & 0.0070 \\
$\Delta \sigma_{8}$ & 0.029 & 0.018 & 0.0042 & 0.076 & 0.080 & 0.0044 \\
$\Delta w_{0}$ & 0.080 & 0.10 & 0.046 & 0.53 & 0.23 & 0.049 \\
$\Delta w_{a}$ & 1.24 & 0.40 & 0.17 & 0.21 & 1.18 & 0.18 \\
$\Delta \Omega_{b} h^{2}$ & 0.0010 & 0.032 & 0.0010 & 1.0 & 0.020 & 0.0010 \\
$\Delta n_{s}$ & 0.040 & 0.12 & 0.025 & 0.43 & 0.041 & 0.014 \\
\hline
\end{tabular}

used. The shear-shear correlations utilize structure information within the redshift range of $[0,3.2]$, while cluster power spectrum survey utilizes only that in the redshift range of $[0.1,1.4]$ (few clusters can be detected at redshifts beyond this range, see 20]). In Table VIII, we show the constraints as in Table I except that we have used only the first four redshift bins for the shearshear correlations, that is, only the source galaxies with redshift $[0,1.6]$ are considered. The Table shows that the constraints would typically degrade by $\sim 30 \%$ if the high$z$ tail of galaxies were discarded. We conclude that about $\sim$ half of the advantage of the shear-shear correlations over the cluster power spectrum comes from this high$z$ tail; the rest of the improvement is due to the fact that at low redshift, the shear-shear correlations (with $\ell_{\max }=1000$ corresponding to $k \sim 1$ at $z \sim 0.2$ ), probe smaller spatial scales than we used for the power spec$\operatorname{trum}\left(k_{\max }=0.15 \mathrm{Mpc}^{-1}\right)$.

\section{E. The Impact of Systematic Errors}

The above results (listed in Table I) are encouraging, and suggest that cluster counts will be a useful com- plement to the shear-shear correlations, despite the fact that constraint from the counts alone are weaker. However, a general concern with this conclusion is that systematic errors, which will inevitably degrade constraints from individual observables, may additionally degrade their synergy. Here we briefly examine some aspects of this question.

First, the major concern with selecting clusters from their weak lensing shear alone is that projection effects will produce false detections (contamination) and cause real clusters to drop out of the sample (incompleteness). 18, 19, 49] In principle, these effects can be modeled in ab-initio simulations, but for a very large survey, such as LSST, the contamination and completeness has to be quantified to a very stringent fractional accuracy of $N_{\text {total }}^{-1 / 2} \sim 2 \times 10^{-3}$ in order not to dominate Poisson errors. Alternative approaches would be to utilize other (optical or X-ray) data to improve the accuracy of the selection function (see ref. 20] for more discussion).

Here we note that if we restrict our analysis to increasingly high $-\sigma$ shear peaks, our results should become increasingly realistic. This is for two reasons: (i) contamination and completeness improve rapidly as the detection threshold (or cluster mass) is increased, 18, 19, 49] and (ii) these peaks are rare, and therefore the required $N_{\text {total }}^{-1 / 2}$ accuracy for the selection function becomes less stringent, and easier to achieve in simulations. Here we simply examine the effect of increasing the threshold $\kappa_{\mathrm{th}}$, to quantify whether a smaller cluster sample, derived from higher shear peaks, is still useful. In Table IX, we repeat our calculations from Table【 except that we replace the threshold $\kappa_{\text {th }}=4.5 \sigma_{\text {noise }}$ by $\kappa_{\text {th }}=10,20,30 \sigma_{\text {noise }}$. The table shows that the number of clusters diminish rapidly: $N_{\text {total }}=276,794 \rightarrow 30,554 \rightarrow 1,954 \rightarrow 205$, respectively, as the threshold is increased. On the other hand, despite this decrease, cluster counts remain useful in tightening the constraints. For example, the most massive $\sim 30,000$ clusters still improve dark energy constraints by a factor of two relative to using the shear-shear correlations alone. Even the most massive $\approx 200-2,000$ clusters, which, by themselves, do not offer interesting constraints on dark energy, still improve the constraints when added to the shear-shear correlations. This gives us confidence that cluster counts will be a useful complement to the shear-shear correlations, despite systematic errors in the weak lensing cluster selection function.

Another significant concern is that clusters are not spherical structures, and, when viewed from different directions, will produce a different shear. Simulations suggest that this can cause an irreducible scatter, and possibly a bias, in the relation between halo mass and shear [50, 51, 52] In addition, fluctuations caused by large-scale structure along the line of sight will introduce a scatter. While once again these effects can be studied in ab-initio simulations, and may be correctable statistically [53] or by identifying foreground lensing galaxies and directly subtracting their lensing effect [54], the ac- 
TABLE IX: This table examines raising the shear-detection threshold. Marginalized errors are shown on cosmological parameters, as in Table [1] except that we adopt increasingly more stringent detection thresholds for the convergence. Note that cluster counts improve dark energy constraints when added to the shear-shear correlations, even for exceedingly high detection thresholds.

\begin{tabular}{|c|c|c|c|c|}
\hline & counts & $\gamma \gamma$ & counts $+\gamma \gamma$ & $\eta$ \\
\hline \multicolumn{4}{|c|}{$\kappa_{\text {th }}=10 \sigma_{\text {noise }}, N_{\text {total }}=30,554$} \\
\hline$\Delta \Omega_{\mathrm{DE}}$ & 0.10 & 0.0084 & 0.0046 & 0.30 \\
$\Delta \Omega_{m} h^{2}$ & 0.35 & 0.049 & 0.0062 & 0.017 \\
$\Delta \sigma_{8}$ & 0.14 & 0.012 & 0.0054 & 0.21 \\
$\Delta w_{0}$ & 0.28 & 0.078 & 0.048 & 0.40 \\
$\Delta w_{a}$ & 2.18 & 0.28 & 0.14 & 0.26 \\
$\Delta \Omega_{b} h^{2}$ & 0.0010 & 0.014 & 0.00099 & 0.99 \\
$\Delta n_{s}$ & 0.040 & 0.050 & 0.017 & 0.30 \\
\hline \multicolumn{5}{|c|}{$\kappa_{\text {th }}=20 \sigma_{\text {noise }}, N_{\text {total }}=1,954$} \\
\hline$\Delta \Omega_{\mathrm{DE}}$ & 0.083 & 0.0084 & 0.0065 & 0.61 \\
$\Delta \Omega_{m} h^{2}$ & 0.71 & 0.049 & 0.0063 & 0.017 \\
$\Delta \sigma_{8}$ & 0.33 & 0.012 & 0.0084 & 0.51 \\
$\Delta w_{0}$ & 2.78 & 0.078 & 0.060 & 0.59 \\
$\Delta w_{a}$ & 6.96 & 0.28 & 0.19 & 0.45 \\
$\Delta \Omega_{b} h^{2}$ & 0.0010 & 0.014 & 0.00099 & 0.99 \\
$\Delta n_{s}$ & 0.040 & 0.050 & 0.017 & 0.31 \\
\hline \multicolumn{5}{|c|}{$\kappa_{\text {th }}=30 \sigma_{\text {noise }}, N_{\text {total }}=205$} \\
\hline$\Delta \Omega_{\mathrm{DE}}$ & 0.52 & 0.0084 & 0.0075 & 0.81 \\
$\Delta \Omega_{m} h^{2}$ & 1.21 & 0.049 & 0.0064 & 0.018 \\
$\Delta \sigma_{8}$ & 0.44 & 0.012 & 0.010 & 0.76 \\
$\Delta w_{0}$ & 12.3 & 0.078 & 0.067 & 0.74 \\
$\Delta w_{a}$ & 39.4 & 0.28 & 0.22 & 0.65 \\
$\Delta \Omega_{b} h^{2}$ & 0.0010 & 0.014 & 0.00099 & 0.99 \\
$\Delta n_{s}$ & 0.040 & 0.050 & 0.018 & 0.33 \\
\hline \multicolumn{5}{|c|}{}
\end{tabular}

curacy to which the magnitude and shape of the unknown scatter will be reduced is not yet clear. Here we perform a simple exercise, and model the probability distribution $p(\kappa \mid M, z)$ for a dark matter halo with fixed mass $M$ at redshift $z$ to produce a smoothed convergence $\kappa$ to be given by a Gaussian,

$$
p(\kappa \mid M, z)=\frac{1}{\sqrt{2 \pi} \sigma_{\kappa}} \exp \left[-\frac{\left(\kappa-\kappa_{\mathrm{NFW}}\right)^{2}}{2 \sigma_{\kappa}^{2}}\right]
$$

where $\kappa_{\mathrm{NFW}}$ is calculated by assuming an NFW density profile for the dark matter halo as described in $\S$. Пabove, and we assume $\sigma_{\kappa}=\epsilon \kappa_{\mathrm{NFW}}$. We assume that the value of $\kappa$ at fixed mass is known ab-initio to within $\sim 30 \%$, i.e we adopt the fiducial value of $\epsilon=0.3$. The probability of detecting this dark matter halo by setting a detection threshold of $\kappa_{\text {th }}$ is then

$$
P(M, z)=\frac{1}{2} \operatorname{erfc}\left[\frac{\kappa_{\mathrm{th}}-\kappa_{\mathrm{NFW}}}{\sqrt{2} \sigma_{\kappa}}\right]
$$

TABLE X: This table examines the effect of scatter in the mass-convergence relation. Marginalized errors are shown on cosmological parameters, as in Table I except that we allow for an additional free parameter, $\epsilon$, representing a scatter between cluster mass and the convergence it produces. We assume a Gaussian distribution for this scatter, and adopt a prior of $\Delta \epsilon=0.3$ when the constraints from the number counts are marginalized over $\epsilon$.

\begin{tabular}{|c|c|c|c|c|c|c|}
\hline & counts & $\gamma \gamma$ & counts $+\gamma \gamma$ & $\eta$ & $\gamma \gamma^{l}$ & counts $+\gamma \gamma^{l}$ \\
\hline$\Delta \Omega_{\mathrm{DE}}$ & 0.11 & 0.0084 & 0.0026 & 0.096 & 0.013 & 0.0028 \\
$\Delta \Omega_{m} h^{2}$ & 0.35 & 0.049 & 0.0061 & 0.016 & 0.034 & 0.0053 \\
$\Delta \sigma_{8}$ & 0.032 & 0.012 & 0.0034 & 0.096 & 0.021 & 0.0052 \\
$\Delta w_{0}$ & 0.077 & 0.078 & 0.032 & 0.35 & 0.12 & 0.035 \\
$\Delta w_{a}$ & 1.32 & 0.28 & 0.11 & 0.17 & 0.42 & 0.14 \\
$\Delta \Omega_{b} h^{2}$ & 0.0010 & 0.014 & 0.00099 & 0.99 & 0.010 & 0.00099 \\
$\Delta n_{s}$ & 0.040 & 0.050 & 0.016 & 0.28 & 0.027 & 0.012 \\
\hline
\end{tabular}

and equation (11) is modified to

$$
N_{i}=\Delta \Omega \Delta z \frac{d^{2} V}{d z d \Omega}\left(z_{i}\right) \int_{0}^{\infty} \frac{d n}{d M}\left(M, z_{i}\right) P\left(M, z_{i}\right) d M .
$$

First, this assumed fiducial scatter increases the number of the total detected clusters (from 276,794 to 305,385). We then recompute the constraints, letting $\epsilon$ to be an additional free parameter, adopting a weak prior of $\Delta \epsilon=$ 0.3. The estimated uncertainties on the cosmological parameters from number counts after marginalizing over $\epsilon$, and its combination with shear-shear correlations, are listed in Table $\mathrm{X}$. A comparison with Table $\square$ reveals that the cluster-count constraints degrade somewhat due to this uncertain scatter, the combined constraint degrade very little. Therefore, we conclude that as long as the $\kappa-M$ relation can be characterized ab-initio to within $\sim 30 \%$, we expect our results to remain realistic.

Another issue is whether photometric redshift accuracies will limit the constraints quoted here. In the case of shear tomography, the impact of redshift uncertainties has been considered in detail in refs [55, 56]. While our redshift bins are relatively wide, calibration of the photometric errors to the accuracy required to avoid degrading dark energy parameter constraints will likely require a large spectroscopic follow-up program. We refer the reader to refs [55, 56] for detailed treatments. In the case of cluster counts, we used 26 redshift bins in our analysis, effectively requiring that we know cluster redshifts to within $\Delta z \approx 0.05$. This is comparable to expected photometric redshift errors, and should be feasible to achieve for most of the clusters, for which a secure identification of cluster membership can be made for a few galaxies. On the other hand, the use of 26 bins is not actually required - one expects that fewer bins are sufficient, since the cluster abundance varies relatively smoothly with redshift.

To address this issue, in Figure 5 we show the marginalized errors on the dark energy parameters from the number counts, as a function of the number of redshift bins 


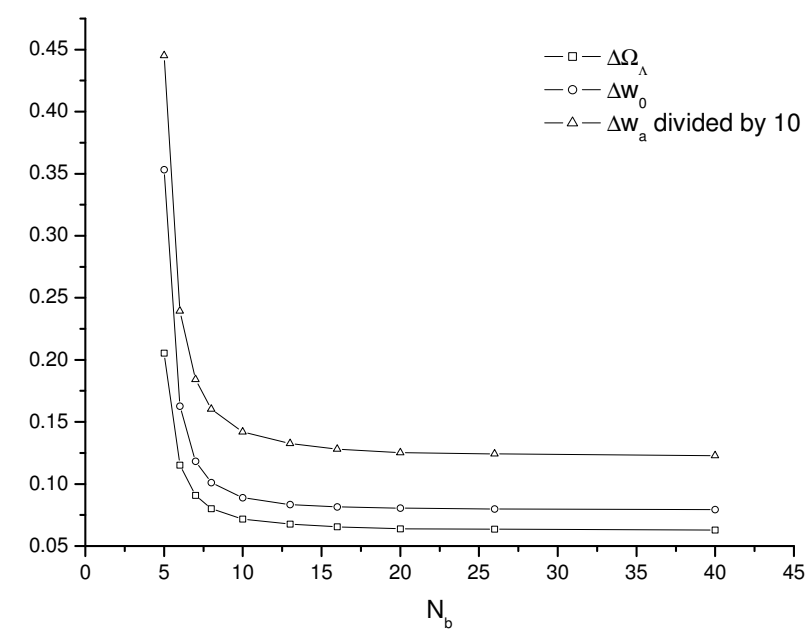

FIG. 5: The marginalized errors are shown on dark energy parameters from the cluster number counts, as a function of the number of redshift bins $\left(N_{b}\right)$ used in the analysis. The bins are assumed to be equally spaced in redshift. The flatness of the curves for $N_{b} \gtrsim 10$ shows that the full information content of the abundance evolution can be extracted with rather modest cluster redshift accuracies of $\Delta z \approx 0.15$.

$N_{b}$. The flatness of the curves on the figure for $N_{b} \gtrsim 10$ shows that the full information content of the abundance evolution can be extracted with rather modest cluster redshift accuracies of $\Delta z \approx 0.15$. This accuracy, however, is still a factor of $\sim 3$ more stringent than the r.m.s. redshift errors expected to be available from tomography alone [19]. Figure 5 shows that with tomographic redshifts alone (i.e. with only $N_{b} \sim 3$ redshift bins), there would be no interesting constraints on dark energy parameters. Hence, to realize the full potential of the survey, it will be important to securely identify mem- ber galaxies in the low-mass clusters and groups at the detection threshold.

\section{CONCLUSIONS}

In this paper, we studied the possibility of improving constrains on dark energy properties by combining two observables - the number counts of detected galaxy clusters and large angular scale tomographic shear-shear correlations - that will both be automatically available in a future large weak lensing survey. We showed that the covariance between these two observables is small, and argued that they can therefore be regarded as independent constraints on dark energy parameters. We used the Fisher matrix formalism to forecast the expected statistical errors from either observable, and from their combination. We found that combining the two observables results in an improvement on dark energy parameter uncertainties by a factor of $2-25$, relative to using either observable alone. We have argued that this conclusion may survive in the face of systematic errors. Our results also suggest that neither observable may exhaust the full information content of a non-linear weak lensing map.

\section{Acknowledgments}

We thank Greg Bryan and Lam Hui for helpful discussions and Sheng Wang and Bhuvnesh Jain for useful comments on the manuscript. This work was supported in part by NSF grant AST-05-07161 (to Z.H.) and by the Initiatives in Science and Engineering (ISE) program at Columbia University.
[1] S. Perlmutter et al., Astrophys. J. 517, 565 (1999).

[2] A. Riess et al., Astron. J. 116, 1009(1998).

[3] S. Dodelson and L. Knox, Phys. Rev. Lett. 84, 3523 (2000).

[4] C. L. Bennett et al., Astrophys. J. Suppl. Ser. 148, $1(2003)$.

[5] Spergel, D. N. et al. 2006, ApJ, in press, astro-ph/0603449

[6] D. Huterer and M. S. Turner, Phys. Rev. D 64, $123527(2001)$.

[7] L. Van Waerbeke and Y. Mellier, astro-ph/0305089.

[8] C.S. Kochanek, P. Schneider, \& J. Wambsganss, in "Gravitational Lensing: Strong, Weak \& Micro.", Lecture Notes of the 33rd Saas-Fee Advanced Course, Eds. G. Meylan, P. Jetzer \& P. North, Springer-Verlag: Berlin, p.273 (2006), in press, astro-ph/0509252

[9] W. Hu, Astophys. J. Lett. 522. L21(1999).

[10] W. Hu, Phys. Rev. D 65, 23003 (2001).
[11] D. Huterer, Phys. Rev. D 65, 63001 (2001).

[12] A. Refregier et al., Astron. J. 127, 3102 (2004).

[13] K. Abazajian and S. Dodelson, Phys. Rev. Lett. 91, 41301 (2003).

[14] M. Takada and B. Jain, Mon. Not. R. Astron. Soc. 348, 897 (2004).

[15] Y.-S. Song and L. Knox, Phys. Rev. D 70, 063510 (2004).

[16] B. Jain, \& L. Van Waerbeke, Astrophys. J. 530, L1 (2000).

[17] S. Wang, Z. Haiman, M. May, \& J. Kehayias, Phys. Rev. D., submitted, astro-ph/0512513

[18] T. Hamana, M. Takada and N. Yoshida, Mon. Not. R. Astron. Soc. 350, 893 (2004).

[19] J. F. Hennawi and D. N. Spergel, Astrophys. J. 624, 59 (2005).

[20] S. Wang, J. Khoury, Z. Haiman and M. May, Phys. Rev. D 70, 123008 (2004).

[21] L. Marian, \& G. M. Bernstein, Phys. Rev. D 73, 123525 
(2006)

[22] L. Wang and P. J. Steinhardt, Astrophys. J. 508, 483 (1998).

[23] Z. Haiman, J. J. Mohr and G. P. Holder, Astrophys. J. 553, 545 (2001).

[24] J. Weller, R. A. Battye and R. Kneissl, Phys. Rev. Lett. 88, 231301 (2002).

[25] J. Weller, R. A. Battye, New Astronomy Reviews 47, 775 (2003).

[26] See www.lsst.org

[27] See pan-starrs.ifa.hawaii.edu

[28] M. S. Turner and M. White, Phys. Rev. D 56, R4439 (1997).

[29] A. Jenkins et al., Mon. Not. R. Astron. Soc. 321, 372 (2001).

[30] J. F. Navarro, C. S. Frenk and S. D. M. White, Astrophys. J. 490, 493 (1997).

[31] G.M. Bernstein, \& M. Jarvis, Astron. J. 123, 583 (2002).

[32] L. Van Waerbeke, Mon. Not. R. Astron. Soc. 313, 524 (2000).

[33] J. L. Tinker, D. H. Weinberg, \& M. S. Warren, Astrophys. J. 647, 737 (2006).

[34] A. Cooray, Mon. Not. R. Astron. Soc. 365, 842 (2006).

[35] C.-P. Ma, R. R. Caldwell, P. Bode and L. Wang, Astrophys. J. 521, L1 (1999).

[36] M. Tegmark, A. N. Taylor and A. F. Heavens, Astrophys. J. 480, 22 (1997).

[37] G. P. Holder, Z. Haiman, J. J. Mohr, Astrophys. J. 560, L111 (2001).

[38] L. Knox, A. Cooray, D. Eisenstein, and Z.Haiman, Astrophys. J. 550, 7 (2001)

[39] R. Scoccimarro, M. Zaldarriaga, and L. Hui, Astrophys.
J. 527, 1 (1999)

[40] P. S. Corasaniti et al. Phys. Rev. D 70, 083006 (2004).

[41] U. Seljak and M. Zaldarriaga, Astrophys. J. 469, 437 (1996). also see www.cmbfast.org

[42] R. E. Smith et al., Mon. Not. R. Astron. Soc. 341, 1311 (2003).

[43] W. Hu, \& A. V. Kravtsov, Astrophys. J. 584, 702 (2003).

[44] M. Lima, \& W. Hu, Phys. Rev. D 70, 043504 (2004).

[45] A. Albrecht et al., astro-ph/0609591.

[46] R.R. Caldwell, R. Dave, \& P. J. Steinhardt, Phys. Rev. Lett, 80, 081582 (1998).

[47] S. Majumdar, \& J.J. Mohr, Astrophys. J. 613, 41 (2004).

[48] W. Hu and Z. Haiman, Phys. Rev. D 68, 3004 (2003)

[49] M. White, L. Van Waerbeke, \& J. Mackey, Astrophys. J. 575, 640 (2002).

[50] R. Cen, Astrophys. J. 485, 39 (1997).

[51] C.A. Metzler, M. White, \& C. Loken, Astrophys. J. 547, 560 (2001).

[52] D. Clowe, A. Gonzalez, \& M. Markevitch, Astrophys. J. 604, 596 (2004).

[53] S. Dodelson, Phys. Rev. D. 70023008 (2004).

[54] R. de Putter, \& M. White, New Astronomy 10, 676 (2005).

[55] Z. Ma, W. Hu \& D. Huterer, Astrophys. J. 636, 21 (2006).

[56] D. Huterer, M. Takada, G. Bernstein, \& B. Jain, Mon. Not. R. Astron. Soc. 366, 101 (2006).

[57] R. K. Sheth and G. Tormen, Mon. Not. R. Astron. Soc. 308, 119 (1999).

[58] B. Jain, U. Seljak, \& S. White, Astrophys. J. 530, 547 (2000).

\section{APPENDIX}

In this Appendix, we give the details of our calculation of the covariance between the cluster counts and the expansion coefficients of the E modes of the shear maps, i.e. equation (29).

\section{Sample Variance of Number Counts}

Following [44], we model the number counts of galaxy clusters in each redshift bin with independent Poisson distributions, whose mean are drawn from correlated Gaussian distributions. Given a cosmological model, the probability density of drawing $\left\{N_{i} ; i=1, \ldots n, n=26\right\}$ clusters, is given by

$$
P\left(\mathbf{N} \mid \overline{\mathbf{N}}, \mathbf{S}^{\text {counts }}\right)=\int d^{n} M\left[\prod_{i=1}^{n} P\left(N_{i} \mid M_{i}\right)\right] G\left(\mathbf{M} \mid \overline{\mathbf{N}}, \mathbf{S}^{\text {counts }}\right)
$$

where $P\left(N_{i} \mid M_{i}\right)$ is the normalized Poisson distribution for $N_{i}$ with a mean $M_{i}$, and $G\left(\mathbf{M} \mid \overline{\mathbf{N}}, \mathbf{S}^{\text {counts }}\right)$ is the normalized multi-variate Gaussian probability distribution for the $M_{i}$ with mean $\overline{\mathbf{N}}$. Here $\mathbf{N}, \overline{\mathbf{N}}$ and $\mathbf{M}$ are 26-dimensional column vectors, representing the counts in the 26 redshift bins, and $\mathbf{S}^{\text {counts }}$ is the $26 \times 26$ covariance matrix. The integral represents averaging over a fluctuating mean $\mathbf{M}$. These fluctuations are due to large-scale fluctuations in the underlying matter density field. Under the assumption that clusters trace the matter density field with a linear bias, we have

$$
M_{i}-\bar{N}_{i}=V_{i} \int d^{3} x W_{i}(\vec{x}(\chi, \hat{n})) b(\chi) \delta(\vec{x}(\chi, \hat{n})) \bar{n}(\chi),
$$

Where we use the comoving radial distance $\chi$ as the time coordinate for the time-dependent quantities, $V_{i}$ is the comoving volume for the $i^{t h}$ redshift bin of cluster counts, $b(\chi)$ is the cluster-averaged linear bias [20, 57], $\delta(\vec{x}(\chi, \hat{n}))$ is the density contrast field of matter, and $W_{i}(\vec{x}(\chi, \hat{n}))$ is the normalized survey window function, which we model to 
be part of a spherical shell. With $W_{i}(\vec{x}(\chi, \hat{n}))=R_{i}(\chi) \Theta(\hat{n})$, we have

$$
\begin{gathered}
R_{i}(\chi)= \begin{cases}3\left[\left(\chi_{\text {max }}^{i}\right)^{3}-\left(\chi_{\text {min }}^{i}\right)^{3}\right]^{-1}, & \chi \in\left[\chi_{\text {min }}^{i}, \chi_{\text {min }}^{i}\right] \\
0, & \text { otherwise }\end{cases} \\
\Theta(\hat{n}(\theta, \varphi))= \begin{cases}{\left[2 \pi\left(1-\cos \theta_{s}\right)\right]^{-1},} & \theta \in\left[0, \theta_{s}\right], \varphi \in[0,2 \pi) \\
0, & \text { otherwise }\end{cases}
\end{gathered}
$$

Where, $\theta_{s}$ is the angular size of the survey region, (in our case, for 18,000 $\operatorname{deg}^{2}$ centered on the pole at $\theta=0$, $\left.\theta_{s}=\arccos \left[1-\frac{5}{18 \pi}\right]\right)$, and $\Delta \chi^{i}\left(=\chi_{\max }^{i}-\chi_{\min }^{i}\right)$ is the comoving radial extent of this $i^{\text {th }}$ redshift bin. $\bar{n}(\chi)$ is the expected comoving number density of the detectable clusters. It is related to $\bar{N}_{i}$ by

$$
\bar{N}_{i}=V_{i} \int d^{3} x W_{i}(\vec{x}(\chi, \hat{n})) \bar{n}(\chi) .
$$

In the limit $\Delta \chi^{i} \rightarrow 0$, we have

$$
R_{i}(\chi) \rightarrow \frac{1}{\chi_{z_{i}}^{2}} \delta\left(\chi-\chi_{z_{i}}\right)
$$

equation (41) reduces to equation (1).

The elements of the sample covariance matrix, $\mathbf{S}^{\text {counts }}$, can be calculated by [43]

$$
S_{i j}^{\text {counts }} \equiv\left\langle\left(M_{i}-\bar{N}_{i}\right)\left(M_{j}-\bar{N}_{j}\right)\right\rangle=\bar{N}_{i} \bar{N}_{j} b\left(z_{i}\right) b\left(z_{j}\right) D\left(z_{i}\right) D\left(z_{j}\right) \sum_{\ell} 4 \pi \tilde{\Theta}_{\ell}{ }^{2} \int \frac{d k}{k} \tilde{R}_{i \ell}(k) \tilde{R}_{j \ell}(k) \Delta^{2}(k, z=0) .
$$

Here $D(z)$ is the growth factor of mass fluctuations, normalized to unity today, $\Delta^{2}(k, z=0)$ is the present-day variance per $\ln k$ for the matter fluctuations, and $\tilde{\Theta}_{\ell}, \tilde{R}_{i \ell}(k)$ are quantities related to the Fourier transform of the survey window function, given by

$$
\tilde{\Theta}_{\ell}= \begin{cases}\sqrt{\frac{1}{4 \pi}}, & \ell=0 \\ \left.\sqrt{\frac{2 \ell+1}{4 \pi}} \frac{(1+x)}{\ell(\ell+1)} \frac{d}{d x} P_{\ell}(x)\right|_{x=\cos \theta_{s},} & \ell \geq 1\end{cases}
$$

and

$$
\tilde{R}_{i \ell}(k) \simeq \frac{1}{\Delta \chi^{i}} \int_{\chi_{\min }^{i}}^{\chi_{\max }^{i}} d \chi j_{\ell}(k \chi),
$$

with $P_{\ell}(x)$ the $\ell^{\text {th }}$ order Legendre polynomials, and $j_{\ell}(k \chi)$ the $\ell^{\text {th }}$ order spherical Bessel functions.

\section{Sample Variance of Shear}

Under the Gaussian assumption for the E mode of the shear field, the probability of drawing a set of spherical expansion coefficients $a_{\ell m}^{b}$ in a given cosmological model is

$$
P\left(\mathbf{a} \mid \mathbf{C}^{\gamma \gamma}\right)=G\left(\mathbf{a} \mid \mathbf{0}, \mathbf{C}^{\gamma \gamma}\right)
$$

where $\mathbf{a}$ is column vector of all the $a_{\ell m}^{b}$ (for our range of $41 \leq \ell \leq 1000$ and with 8 redshift bins, this vector has dimension $\left.8 \times \Sigma_{41}^{1000}(2 \ell+1)=8,002,560\right), \mathbf{0}$ is a column zero vector of the same dimension, and $\mathbf{C}^{\gamma \gamma}=\mathbf{S}^{\gamma \gamma}+\mathbf{N}^{\gamma \gamma}$ is the covariance matrix for the $a_{\ell m}^{b}$, and $\mathbf{S}^{\gamma \gamma}$ and $\mathbf{N}^{\gamma \gamma}$ are as given in Sec. IIB, Note that both $\mathbf{S}^{\gamma \gamma}$ for the shear field and $\mathbf{S}^{\text {counts }}$ for the cluster number counts come from fluctuations in the matter distribution. Since the shear field and the clusters are detected in the same realization of the matter distribution, they can not be independent.

The convergence (or E mode of the shear field) signal from the source galaxies in redshift bin $b$ is calculated by

$$
\kappa^{b}(\hat{n})=\frac{1}{2 c^{2}} \int_{0}^{\chi \infty} d \chi\left(\nabla^{2}-\nabla_{\chi}^{2}\right) \Phi(\vec{x}(\chi, \hat{n})) W^{b}(\chi)
$$


where $\Phi(\vec{x}(\chi, \hat{n}))$ is the gravitational potential field. As discussed in [58], the integral of the term that contains $\nabla_{\chi}^{2} \Phi$ is much smaller than that containing $\nabla^{2} \Phi$ everywhere except on the largest angular scales. We thus neglect this term in the following calculation. Expanding $\kappa^{b}(\hat{n})$ in spherical harmonics, we get

$$
a_{\ell m}^{b}=\int d \Omega \kappa^{b}(\hat{n}) Y_{\ell m}^{*}(\hat{n})+\text { noise. }
$$

The Poisson equation reads as

$$
\nabla^{2} \Phi(\vec{x}(\chi, \hat{n}))=4 \pi G a^{2}(\chi) \rho_{m}(\chi) \delta(\vec{x}(\chi, \hat{n}))
$$

with $\delta(\vec{x}(\chi, \hat{n}))$ in this equation representing the (non-linear) matter density contrast. Using this equation, we find

$$
a_{\ell m}^{b}=\frac{2 \pi G}{c^{2}} \int d \chi a^{2}(\chi) \rho_{m}(\chi) W^{b}(\chi) \int d \Omega Y_{\ell m}^{*}(\hat{n}) \delta(\vec{x}(\chi, \hat{n}))+\text { noise. }
$$

The sample variance $\mathbf{S}^{\gamma \gamma}$ and full covariance $\mathbf{C}^{\gamma \gamma}$ can then be computed as the ensemble average $\left\langle a_{\ell m}^{b} a_{\ell^{\prime} m^{\prime}}^{b^{\prime} *}\right\rangle$ with or without the noise term (the latter leads to equation 26).

\section{Covariance Between Number Counts and Shear}

The correlation between $\left(M_{i}-\bar{N}_{i}\right)$ and $a_{\ell m}^{b}$ is nonzero because they depend on the same density field. For clarity, from now on, we add a prime to the coordinates relating to the calculation of $a_{\ell m}^{b}$. The correlation between the two density contrast fields is given by the two-point function

$$
\left\langle\delta(\vec{x}(\chi, \hat{n})) \delta\left(\overrightarrow{x^{\prime}}\left(\chi^{\prime}, \hat{n}^{\prime}\right)\right)\right\rangle=D(\chi) D\left(\chi^{\prime}\right) \int \frac{d^{3} k}{(2 \pi)^{3}} e^{i \vec{k} \cdot\left(\vec{x}-\vec{x}^{\prime}\right)} P\left(k, \chi_{p}=0\right) .
$$

The covariance of $\left(M_{i}-\bar{N}_{i}\right)$ and $a_{\ell m}^{b}$ is then given by

$$
\begin{array}{r}
\left\langle\left(M_{i}-N_{i}\right) a_{\ell m}^{b}\right\rangle=\frac{2 \pi G}{c^{2}} V_{i} \int \chi^{2} d \chi R_{i}(\chi) b(\chi) \bar{n}(\chi) D(\chi) \int d \chi^{\prime} a^{2}\left(\chi^{\prime}\right) \rho_{m}\left(\chi^{\prime}\right) W^{b}\left(\chi^{\prime}\right) D\left(\chi^{\prime}\right) \\
\int \frac{k^{2} d k}{(2 \pi)^{3}} P\left(k, \chi_{p}=0\right) \int d \Omega_{k} \int d \Omega^{\prime} e^{-i k \chi^{\prime} \hat{k} \cdot \hat{n}^{\prime}} Y_{\ell m}^{*}\left(\hat{n^{\prime}}\right) \int d \Omega e^{i k \chi \hat{k} \cdot \hat{n}} \Theta(\hat{n}) .
\end{array}
$$

Note noise from intrinsic ellipticity of galaxies does not correlate with this fluctuation of cluster counts, so the noise term drops here. The integral over $\Omega$ gives

$$
I_{\Omega}=\sum_{\ell^{\prime}=0}^{\infty} \sum_{m=-\ell^{\prime}}^{\ell^{\prime}} 4 \pi i^{\ell^{\prime}} j_{\ell^{\prime}}(k \chi) Y_{\ell^{\prime} m^{\prime}}(\hat{k}) \tilde{\Theta}_{\ell^{\prime}} \delta_{m^{\prime} 0},
$$

and the integral over $\Omega^{\prime}$ gives

$$
I_{\Omega^{\prime}}=4 \pi(-i)^{\ell} Y_{\ell m}^{*}(\hat{k}) j_{\ell}\left(k \chi^{\prime}\right) .
$$

We are now ready to calculate the integral over $\Omega_{k}$, which gives

$$
I_{\Omega_{k}}=\delta_{m 0}(4 \pi)^{2} \tilde{\Theta}_{\ell} j_{\ell}(k \chi) j_{\ell}\left(k \chi^{\prime}\right) .
$$

We next use the Limber approximation when integrating over $k$. Assuming that $P\left(k, \chi_{p}=0\right)$ is a slowly varying function compared to the $j_{\ell}(k \chi)$, we have

$$
I_{k} \simeq \delta_{m 0} \tilde{\Theta}_{\ell} \frac{1}{\chi^{2}} \delta\left(\chi-\chi^{\prime}\right) P\left(k=\frac{\ell}{\chi}, \chi_{p}=0\right),
$$

where we have used the orthogonality property

$$
\int_{0}^{\infty} k^{2} d k j_{\ell}(k \chi) j_{\ell}\left(k \chi^{\prime}\right)=\frac{\pi}{2 \chi^{2}} \delta\left(\chi-\chi^{\prime}\right)
$$


for $\ell>0$. The integral over $\chi^{\prime}$ is easy to perform, and we finally have

$$
\left\langle\left(M_{i}-N_{i}\right) a_{\ell m}^{b}\right\rangle=\frac{2 \pi G}{c^{2}} \delta_{m 0} V_{i} \tilde{\Theta}_{\ell} \int d \chi R_{i}(\chi) b(\chi) \bar{n}(\chi) a^{2}(\chi) \rho_{m}(\chi) W^{b}(\chi) P\left(k=\frac{\ell}{\chi}, \chi\right) .
$$

recalling the evolution of the matter density,

$$
\rho_{m}(\chi)=\Omega_{m} \frac{3 H_{0}^{2}}{8 \pi G} \frac{1}{a(\chi)^{3}},
$$

and of the power spectrum,

$$
\Delta^{2}(k, \chi)=\frac{k^{3}}{2 \pi^{2}} P(k, \chi)
$$

by taking the limit of $\Delta \chi^{i} \rightarrow 0$, we obtain the final result.

$$
\left\langle\left(M_{i}-N_{i}\right) a_{\ell m}^{b}\right\rangle=\delta_{m 0} \frac{3 \pi^{2}}{2} \frac{\Omega_{m} H_{0}^{2}}{c^{2} a\left(z_{i}\right)} \frac{\chi_{z_{i}}}{\ell^{3}} \tilde{\Theta}_{\ell} N_{i} b\left(z_{i}\right) W^{b}\left(z_{i}\right) \Delta^{2}\left(k=\frac{\ell}{\chi_{z_{i}}}, z_{i}\right) .
$$

This result is quoted above in equation (29), and the corresponding cross-correlation coefficient is evaluated explicitly in one example in Figure 1 .

Given the above result on the cross correlation, we can write down the expression for the joint probability distribution for simultaneously drawing a set of $N_{i}$ and $a_{\ell, m}^{b}$,

$$
P\left(\mathbf{N}, \mathbf{a} \mid \overline{\mathbf{N}}, \mathbf{C}_{\mathbf{t o t}}\right)=\int d^{n} M\left[\prod_{i=1}^{n} P\left(N_{i} \mid M_{i}\right)\right] G\left(\mathbf{T} \mid \overline{\mathbf{T}}, \mathbf{C}_{\mathbf{t o t}}\right)
$$

where $\mathbf{T}$ is a column vector that combines $\mathbf{M}$ and $\mathbf{a} ; \overline{\mathbf{T}}$ is the column vector containing the mean values $\overline{\mathbf{N}}$ and $\mathbf{0}$; and $\mathbf{C}_{\text {tot }}$ is the total covariance matrix, consisting of four blocks,

$$
\mathbf{C}_{\text {tot }}=\left(\begin{array}{cc}
\mathbf{S}^{\text {counts }} & \mathbf{C}^{\text {cross }} \\
\left(\mathbf{C}^{\text {cross }}\right)^{\mathrm{T}} & \mathbf{C}^{\gamma \gamma}
\end{array}\right)
$$

Note that here $\mathbf{C}^{\gamma \gamma}$ is the full shear-covariance matrix, including the shear noise (in practice, we found shear noise to have only a small effect on the results). When the $\xi_{i, b \ell m}$ are small, we can write the multi-variate Gaussian as a product of two independent Gaussians,

$$
G\left(\mathbf{T} \mid \overline{\mathbf{T}}, \mathbf{C}_{\text {tot }}\right)=G\left(\mathbf{M} \mid \overline{\mathbf{N}}, \mathbf{S}^{\text {counts }}\right) G\left(\mathbf{a} \mid \mathbf{0}, \mathbf{C}^{\gamma \gamma}\right)+o(\xi),
$$

and the joint probability becomes separable,

$$
P\left(\mathbf{N}, \mathbf{a} \mid \overline{\mathbf{N}}, \mathbf{C}_{\text {tot }}\right)=P\left(\mathbf{N} \mid \overline{\mathbf{N}}, \mathbf{S}^{\text {counts }}\right) P\left(\mathbf{a} \mid \mathbf{C}^{\gamma \gamma}\right)+o(\xi),
$$

justifying the assumption that cluster number counts and shear-shear correlations can be treated as independent cosmological probes. 